\title{
Aufsatz
}

Christian Koller

\section{The British Foreign Legion - Ein Phantom zwischen Militärpolitik und Migrationsdiskursen}

DOI 10.1515/mgzs-2015-0002

Zusammenfassung: Im Unterschied zu Frankreich und einigen anderen Kolonialmächten hat das Vereinigte Königreich nie über eine permanente Fremdenlegion verfügt. Ansätze dazu bestanden indessen sehr wohl. Für den Krimkrieg wurde zwar eine britische Fremdenlegion rekrutiert, diese kam jedoch nicht zum Einsatz. Teile dieser Truppe gelangten später als Militärsiedler nach Südafrika. Vom Ersten Weltkrieg bis in den frühen Kalten Krieg gab es dann in Politik und Presse immer wieder Stimmen, die eine Fremdenlegion nach französischem Vorbild forderten, um Exilanten der Krone nutzbar zu machen. Der Artikel diskutiert diese im Schnittpunkt von Militär- und Migrationsgeschichte stehenden Ansätze zur Etablierung einer britischen Fremdenlegion im Kontrast zur französischen Entwicklung und analysiert, welche Einflussfaktoren $\mathrm{zu}$ bestimmten Zeitpunkten Forderungen nach Einrichtung einer britischen Fremdenlegion haben laut werden lassen und welche die dauerhafte Gründung einer solchen Institution schließlich verhinderten.

Schlüsselwörter: Söldnertum, Migration, Großbritannien, Flüchtlinge, Militärpolitik

Im November 2005 überschrieb die Londoner »Times« einen Artikel über aktuelle Entwicklungen in den Streitkräften Ihrer Majestät mit dem reißerischen Titel: »How British Army is fast becoming foreign legion «" ${ }^{1}$ Der Artikel führte aus, dass wegen eines massiven Anstiegs ausländischer Soldaten, deren Zahl sich seit 1998, als die Restriktionen bei der Rekrutierung von Bewerbern aus den Com-

1 Times, 14.11.2005. Ähnlich titelte die »Sun« am 27.12.2007 (»UK Army is a foreign legion«) und 30.9.2012 (»British `foreign legion« trebles in decade«).

Kontakt: Christian Koller, Universität Zürich, Schweizerisches Sozialarchiv, E-Mail: koller@sozarch.uzh.ch 
monwealth-Staaten gelockert worden waren, etwa verdreißigfacht hätte, eine Begrenzung angestrebt werde. Insgesamt machten diese Soldaten im Jahre 2005 sechs Prozent des gesamten Mannschaftsbestands der britischen Armee aus, zu denen noch weitere drei Prozent an nepalesischen Gurkhas kamen. Der Artikel endete mit einem Infokasten, der unter dem Titel »The French Way« in knapper Form die wichtigsten Fakten zur gegenwärtigen französischen Fremdenlegion auflistete.

Die Rekrutierung von Ausländern erschien hier also als etwas typisch Französisches. Dies zeugt vom anhaltenden Bekanntheitsgrad der 1831 gegründeten Fremdenlegion, deren Ruf als eine der härtesten Truppen der Welt, aber auch als Sammelbecken von Abenteurern, Aussteigern und Kriminellen seit dem 19. Jahrhundert ambivalent ist. Effektiv rekrutierte sich die "Légion étrangère«, die bis 1962 eine wichtige Stütze des französischen Kolonialimperialismus darstellte, im Wesentlichen aus zwei Gruppen: arbeits- und perspektivlosen Angehörigen der europäischen Unterschichten und politischen Flüchtlingen ${ }^{2}$. Auch andere Kolonialmächte bedienten sich vergleichbarer Einheiten. Die Niederlande rekrutierten von 1830 bis 1909 zahlreiche Ausländer für die »Koninklijk Nederlandsch-Indische Leger « zur Sicherung von Niederländisch-Ostindien, und 1920 wurde nach dem französischen Vorbild eine spanische Fremdenlegion (»Tercio de Extranjeros«) ins Leben gerufen, die indessen immer einen großen Anteil von Spaniern aufwies und nach mehreren Namenswechseln schließlich zu »La Legión« wurde³.

Großbritannien dagegen rang sich nie zur dauerhaften Einrichtung einer Fremdenlegion durch. Dies erstaunt umso mehr, als das Vereinigte Königreich beim Übergang von den frühneuzeitlichen Söldnerheeren $\mathrm{zu}$ den modernen Nationalarmeen im Unterschied zu seinen europäischen Rivalen auf die Einführung der allgemeinen Wehrpflicht verzichtete und sich stattdessen auf eine Berufsarmee sowie zahlreiche in den Kolonien selbst rekrutierte Streitkräfte stützte. Sarah Percy hat diese Sonderentwicklung damit zu erklären versucht, dass in Großbritannien zwei starke Normen bestanden hätten: Abneigungen gegen eine stehende Armee und gegen Söldner ${ }^{4}$. Letztere manifestierte sich etwa in der internen Kritik am Einsatz deutscher Söldner während des amerikanischen Unabhängigkeitskrieges ${ }^{5}$.

2 Vgl. Christian Koller, Die Fremdenlegion. Kolonialismus, Söldnertum, Gewalt, 1831-1962, Paderborn [u. a.] 2013, S. 39-50.

3 Vgl. John Scurr, The Spanish Foreign Legion, London 1985; José Luis Rodríguez Jiménez, iA mí la Legión! De Millán Astray a las misiones de paz, Barcelona 2005.

4 Sarah Percy, Mercenaries. The History of a Norm in International Relations, Oxford 2007, S. $148-163$.

5 Vgl. z.B. The Parliamentary History of England, from the earliest period to the year 1803, vol. 19, London 1814 (Ndr. New York 1966), S. 371. 
Immerhin gab es im 19. und 20. Jahrhundert aber verschiedentlich Ansätze oder zumindest Diskussionen zur Etablierung einer britischen Fremdenlegion, die indessen nie zu einem dauerhaften Resultat führten. Diese Ansätze und Diskussionen sollen im Folgenden erstmals im Zusammenhang und im Kontrast zur französischen Entwicklung diskutiert werden. Insbesondere gilt es $\mathrm{zu}$ analysieren, welche Einflussfaktoren $\mathrm{zu}$ bestimmten Zeitpunkten Forderungen nach Einrichtung einer britischen Fremdenlegion haben laut werden lassen und welche die dauerhafte Gründung einer solchen Institution schließlich verhinderten. Der Fokus liegt dabei auf dem Zusammenspiel von militär- und migrationspolitischen Faktoren beziehungsweise Angebot und Nachfrage nach (primär europäischen) Söldnern.

Ich werde nach einem knappen Überblick über das Söldnerwesen seit 1800, als die vormodernen Söldnerheere nach landläufiger Ansicht von den modernen Nationalarmeen verdrängt wurden, in einem ersten Abschnitt Ansätze zu einer britischen Fremdenlegion im 19. Jahrhundert untersuchen. Der zweite Abschnitt befasst sich mit den einschlägigen Diskussionen im Zeitalter der Weltkriege, die nie zu einem konkreten Resultat führen sollten. Der dritte Abschnitt konzentriert sich dann auf Forderungen nach einer britischen Fremdenlegion im frühen Kalten Krieg und gibt auch einen Ausblick auf die Entwicklung bis in die Gegenwart.

\section{Söldnertum im Zeitalter der Nationalarmeen}

Entgegen der gängigen Vorstellung, das Söldnerwesen sei Ende des 18. Jahrhunderts zusammen mit dem »Ancien Régime« untergegangen und habe einem Zeitalter der auf der allgemeinen Wehrpflicht beruhenden Nationalarmeen Platz gemacht, existierten im 19. Jahrhundert noch zahlreiche Söldnerarmeen, die sich grob in drei Sphären einteilen lassen: Erstens bestand das klassische europäische Söldnerwesen an gewissen Orten noch bis weit ins 19. Jahrhundert hinein fort (etwa im Königreich beider Sizilien bis 1859 und im Kirchenstaat bis 1870). Zweitens wurden Kolonialkriege in aller Regel nicht mit Wehrpflichtigen geführt, sondern mit Berufssoldaten aus der Imperialmacht, afrikanischen und asiatischen Kolonialeinheiten sowie aus europäischen Söldnern bestehenden Truppen. Und drittens erfolgten praktisch bei jedem bewaffneten Konflikt in Lateinamerika Truppenanwerbungen in Europa und rekrutierten Staaten wie Argentinien zuweilen auch Wehrbauern zur Peuplierung und militärischen Absicherung dünn besiedelter Gebiete. Es bestand also ein beinahe globaler Söldnermarkt mit einer Vielzahl von Arbeitgebern in drei Sphären, zwischen denen die Übergänge sowohl strukturell als auch individuell fließend waren. 
Die Geschichte der modernen britischen und französischen Fremdenlegionen begann in den 1830er Jahren, es gab indessen Vorläufer. Beide Länder hatten in der frühen Neuzeit Gebrauch von Söldnern gemacht, die direkten Vorläufer sind aber in der Umbruchszeit um 1800 zu verorten. Auf der britischen Seite wurden ab 1794 in den Kriegen gegen das revolutionäre Frankreich in großer Zahl Hannoveraner, Hessen, Badenser und französische Exilanten eingesetzt, und von 1803 bis 1816 existierte eine »King’s German Legion«, die nach der Auflösung des mit der britischen Krone in Personalunion verbundenen Kurfürstentums Hannover gebildet und 1816 in die Armee des neu geschaffenen Königreichs Hannover überführt wurde $^{6}$. In Frankreich entstand 1815/16 aus einem Teil der napoleonischen Fremdenregimenter das Regiment Hohenlohe (bis 1821 »Légion étrangère royale«), das im Zuge der Julirevolution 1830 aufgelöst wurde, dessen Soldaten aber (ebenso wie diejenigen der ebenfalls aufgelösten königlichen Schweizerregimenter) eine Rekrutierungsbasis für die neue Fremdenlegion bildeten ${ }^{7}$. Im Unterschied zu Großbritannien gab es also in Frankreich bereits vor der Gründung der modernen Fremdenlegion eine weit stärkere Tendenz zur Kontinuität, die dem säkularen Trend zur Einschränkung des Söldnertums widerstand.

Im 20. Jahrhundert verengte sich das europäische Söldnerwesen dann im Wesentlichen auf die französische und in geringerem Ausmaß die spanische Fremdenlegion. Während diese die Rekrutierung von Ausländern immer mehr einschränkte, baute jene im Zeitalter der Welt- und dann der Dekolonisationskriege ihre Bestände stark aus, um sich nach der Unabhängigkeit Algeriens 1962 radikal zu verkleinern. Danach verlagerte sich der globale Schwerpunkt des Söldnerwesens nach Afrika mit seinen zahlreichen Bürgerkriegen, in denen teilweise auch Europäer und Amerikaner zum Einsatz gelangten ${ }^{8}$. Das Aufkommen von Militärunternehmen nach dem Ende des Kalten Krieges führte dann zu einer Renaissance des Söldnerwesens in privatisierter Form ${ }^{9}$. Auch in Großbritannien entstand eine

6 C[harles] C. Bayley, Mercenaries for the Crimea. The German, Swiss, and Italian Legions in British Service, 1854-1856, Montreal 1977, S. 7; North Ludlow Beamish, History of the King's German Legion, 2 vols., London 1832-1837; Mike Chappell, The King's German Legion, 2 vols., Oxford 2000; Brendan Simms, The longest afternoon: The 400 men who decided the battle of Waterloo, London 2014.

7 Douglas Porch, The French foreign legion. A complete history, New York, London 1991, S. 1-11; Jérôme Bodin, Les Suisses au service de la France. De Louis XI à la Légion étrangère, Paris 1988, S. $295-328$.

8 Vgl. z.B. Scott Fitzsimmons, Mercenaries in Asymmetric Conflicts, Cambridge 2012.

9 Vgl. z.B. Peter W. Singer, Corporate Warriors. The Rise of the Privatized Military Industry, Ithaca, NY 2003; Chris Kinsey, Corporate Soldiers and International Security. The Rise of Private Military Companies, London [u.a.] 2006; Laurent Joachim, Der Einsatz von »Private Military Companies« im modernen Konflikt. Ein neues Werkzeug für »Neue Kriege«?, Berlin 2010. 
Reihe solcher Unternehmen, so als Vorreiter bereits 1965 »Watch Guard International«, 1995 »Sandline International« oder 2002 die »Aegis Defence Services«. 2006 wurde mit der »British Association of Private Security Companies« (BAPSC) gar ein Branchenverband ins Leben gerufen. Bezeichnenderweise ratifizierte bislang kein einziges permanentes Mitglied des Weltsicherheitsrates die 1989 von der UNO-Generalversammlung beschlossene und 2001 in Kraft getretene Konvention gegen Rekrutierung, Verwendung, Finanzierung und Ausbildung von Söldnern.

Parallel zum Söldnertum und mit diesem teilweise strukturell wie individualbiografisch überlappend entwickelte sich im 19. und 20. Jahrhundert der hauptsächlich ideologisch motivierte fremde Kriegsdienst. Bei zahlreichen bewaffneten Konflikten, die irgendwie den Charakter von »Befreiungskriegen« trugen, gab es im 19. Jahrhundert internationale Freiwilligenverbände (etwa Bolivars britische Legion von 1819 bis 1824 oder Garibaldis Internationale Legion von 1860). Die bekanntesten Beispiele aus dem 20. Jahrhundert sind die Internationalen Brigaden des Spanischen Bürgerkrieges und die ausländischen Verbände der WaffenSS. Der Charakter der französischen Fremdenlegion näherte sich in den Ausnahmesituationen der beiden Weltkriege solchen »ideologischen« Truppen an, als Freiwillige, die aus politischer Sympathie für Frankreich kämpfen wollten, formell zu Fremdenlegionären wurden ${ }^{10}$. Auch britische und amerikanische Pläne im frühen Kalten Krieg für die Aufstellung antikommunistischer Söldnertruppen zielten, wie noch zu zeigen sein wird, in diese Richtung.

\section{Britische »Foreign Legions« im 19. Jahrhundert}

Bereits in der Gründungsphase der 1830er Jahre zeigten sich zwischen dem französischen und dem britischen Ansatz markante Unterschiede. Die französische Fremdenlegion wurde 1831 mit dem hauptsächlichen Ziel gegründet, nebst den Söldnern der aufgelösten königlichen Fremdenregimenter auch die aus verschiedenen Ländern, insbesondere aus Polen, nach Frankreich strömenden Flüchtlinge der 1830er-Erhebungen außer Landes zu schaffen und sie für die unter der vorangegangenen Regierung angelaufene Eroberung Algeriens nutzbar zu machen. Die neue Regierung des »Bürgerkönigs « glaubte dadurch zwei Fliegen mit einer Klappe schlagen zu können, nämlich als potenzielle Unruhestifter betrachtete Personen loszuwerden, ohne ihr liberales Image zu beschädigen - in den liberalen Kreisen

10 Vgl. Pierre Dufour, La légion en 14-18, Paris 2003; Pierre Dufour, La Légion Etrangère 1939-1945, Bayeux 2000; Martin Windrow, French Foreign Legion, 1914-1945, Oxford 1999; Jérôme Christinaz, Le recrutement des étrangers à Paris en août 1914. Le corps des volontaires suisses. Lizentiatsarbeit Université de Fribourg 2010. 
Europas herrschte nach 1830 eine große Polenbegeisterung vor ${ }^{11}-$, und zugleich das Leben eigener Landessöhne in einer wenig populären Militärexpedition zu schonen. Die Integration zahlreicher polnischer Flüchtlinge in die Fremdenlegion erregte denn auch die Aufmerksamkeit und teilweise Kritik der europäischen liberalen Presse ${ }^{12}$.

Im Jahre 1835 überließ Frankreich seine gesamte Fremdenlegion per Vertrag der spanischen Regierung unter Isabella II., die sich in einem bis 1839 dauernden Thronfolgekrieg gegen die Karlisten befand. In diesem Bürgerkrieg kam die erste Generation von Fremdenlegionären fast vollumfänglich ums Leben ${ }^{13}$. Ironischerweise sollten die Reihen der Fremdenlegion in der Folge wesentlich durch nach Frankreich geflohene Karlisten wieder aufgefüllt werden ${ }^{14}$. Obwohl die endgültige Verstetigung der französischen Fremdenlegion erst mit dem Krimkrieg angesetzt werden kann, existierte also bereits im ersten Vierteljahrhundert nach 1831 permanent eine Söldnertruppe unter dieser Bezeichnung.

Ein vergleichbarer, auch auf die Militärverfassung durchschlagender Umbruch wie die Julirevolution fehlte in Großbritannien. Auch die liberale Regierung Palmerston unterstützte indessen die spanische Königin Isabella und stellte dazu eine »British Auxiliary Legion« in deren Dienste ${ }^{15}$. Zwar wurde diese Einheit zwei

11 Vgl. z.B. Gabriela Brudzyńska-Němec, Polenvereine in Baden. Hilfeleistung süddeutscher Liberaler für die polnischen Freiheitskämpfer 1831-1832, Heidelberg 2006; Der polnische Freiheitskampf 1830/31 und die liberale deutsche Polenfreundschaft. Hrsg. von Peter Ehlen, München 1982; Anneliese Gerecke, Das deutsche Echo auf die polnische Erhebung von 1830, Wiesbaden 1964; Jan Kosim, Zur Geschichte der Vereine zur Unterstützung der Polen in Deutschland unter Berücksichtigung der Bayerischen Pfalz und der Landgrafschaft Hessen-Homburg. In: Jahrbuch für westdeutsche Landesgeschichte, 4 (1978), S. 313-355.

12 Vgl. z.B. Neue Zürcher Zeitung, 30.10.1833; St. Galler Zeitung, 19.9.1835; Journal de Genève, 14.4.1836; Gazette de Lausanne, 29.4.1836.

13 Vgl. Paul Azan, La Légion Etrangère en Espagne 1835-1839, Paris 1906; Porch, The French foreign legion (wie Anm. 7), S. 22-49; Pierre Montagnon, La Légion étrangère. De 1831 à nos jours, Paris 1999, S. 20-28.

14 St. Galler Zeitung, 5.8.1840.

15 Vgl. z.B. Morning Chronicle, 3.7.1835, 21.8.1837; Times, 5.10.1835, 21.9.1837, 18.11.1837, 15.12.1837; Caledonian Mercury, 12.10.1835, 25.7.1836; Manchester Guardian, 14.2.1836, 15.2.1836; North Wales Chronicle, 23.2.1836; The Standard, 7.3.1836; Morning Post, 18.4.1836, 28.8.1837, 21.9.1837, 27.9.1837, 15.1.1838, 10.2.1838; Twelve Months in the British Legion. By an officer of the ninth regiment, London 1836; A concise account of the British auxiliary legion commanded by General Evans, Scarborough 1837; J.H. Humphrey, A Concise Review of the Campaigns of the British Legion in Spain. With a Full Statement of the Grounds in Which Their Claims on the British Government are Based and A View of the Political Question Which Originated the Expedition, London 1838; Alexander Somerville, A narrative of the British Auxiliary Legion, with incidents, anecdotes and sketches of all parties connected with the war in Spain, from a journal of personal observations, Glasgow 1838; Edward M. Spiers, Radical general. Sir George de Lacy Evans 
Jahrzehnte später in einer Zusammenstellung des »War Department« über die britischen »Foreign Legions« als »Spanish Contingent « zusammen mit den Fremdenlegionen des Krimkrieges aufgelistet ${ }^{16}$, die Ausländer waren darin aber in der Minderheit. Die Ex-post-Bezeichnung »Foreign Legion« meinte also eine in ausländischen Diensten stehende, nicht eine aus Ausländern bestehende Einheit. Zeitgenössisch wurde sie in der Presse nebst der offiziellen Titulatur auch alternativ »Spanish Mercenaries « oder »British Mercenaries in Spain « genannt ${ }^{17}$. Effektiv bestand die unter dem Kommando von Sir George De Lacy Evans stehende Truppe hauptsächlich aus britischen und irischen Freiwilligen, darunter vielen Armeeangehörigen und Angehörigen der ländlichen Unterschichten, die durch die »enclosures « sozial abgestiegen waren ${ }^{18}$. Voraussetzung für die Rekrutierung der Legion war denn auch eine Suspension des »Foreign Enlistment Bill« am 10. Juni 1835, der für alle britischen Untertanen den Kriegsdienst für eine fremde Macht an die Zustimmung der Krone knüpfte ${ }^{19}$.

Die »Auxiliary Legion« rekrutierte aber, hierin der französischen Fremdenlegion ähnlich, auch eine unbekannte Anzahl von Exilpolen, von denen einige bereits in napoleonischen Diensten gestanden hatten und für die eine eigene kleine Einheit gebildet wurde. Bei ihrer Anwerbung zahlte das »Committee for the Relief of Polish Exiles« acht Shilling pro Person in die Kasse der Legion, »with the understanding that hereafter they renounce all further claims upon its assistance $\aleph^{20}$. Ferner taten weitere Ausländer in der »Auxiliary Legion« Dienst, darunter Baron Richard von Stutterheim, der wegen eines Duells den preußischen Dienst verlassen hatte und später im Krimkrieg die »British German Legion« kommandieren sollte ${ }^{21}$. Die meisten Überlebenden der anfangs etwa 10000 Freiwilligen quittierten nach Ablauf ihres Zweijahreskontraktes den Dienst. Eine 1837 rekrutierte, weit kleinere »New Legion« wurde bereits im Jahr darauf wieder aufgelöst ${ }^{22}$.

1787-1870, Manchester 1983, S. 66-100; Edward M. Brett, The British Auxiliary Legion in the first Carlist War in Spain, 1835-1838, A forgotten army, Dublin 2005.

16 Vgl. The National Archives (TNA), WO 25/3861: Foreign Legions.

17 Morning Post, 28.9.1835, 2.10.18352, 23.7.1836.

18 Vgl. Martin Robson, »Strangers, Mercenaries, Heretics, Scoffers, Polluters«. Volunteering for the British Auxiliary Legion in Spain, 1835. In: Transnational Soldiers. Foreign Military Enlistment in the Modern Era. Ed. by Nir Arielli and Bruce Collins, Basingstoke 2013, S. 181-201; Brett, The British Auxiliary Legion (wie Anm. 15), S. 31-46. Eine Namensliste der Legion ist für die Mannschafts- und Unteroffizierränge nicht erhalten (ebd., S. 3).

19 Brett, The British Auxiliary Legion (wie Anm. 15), S. 25 f.

20 Blackburn Standard, 29.7.1835.

21 Vgl. Bernhard von Poten, »Stutterheim, Richard von«. In: Allgemeine Deutsche Biographie, 37 (1894), S. $79 \mathrm{f}$.

22 Brett, The British Auxiliary Legion (wie Anm. 15), S. 150-162. 
Während die französische Regierung 1836 eine »Nouvelle Légion« aufstellte, um den weiterhin vor allem aus Polen nach Frankreich strebenden Flüchtlingsstrom zu kanalisieren und Einheiten für die weitere Eroberung Algeriens zu gewinnen ${ }^{23}$, sah man in Großbritannien zu einem solchen Schritt weder militär- und kolonialnoch flüchtlingspolitisch eine Notwendigkeit.

Eine mit der französischen Fremdenlegion vergleichbare und auch so benannte Einheit wurde dann knapp zwei Jahrzehnte darauf für das britische Eingreifen in den Krimkrieg aufgestellt ${ }^{24}$. Dabei spielten die Nachfrage nach rasch verfügbaren Söldnern mit dem Vorhandensein einer großen Zahl perspektivloser Flüchtlinge im Gefolge der 1848er-Revolutionen zusammen. Im Unterschied zu Frankreich mit seiner Wehrpflichtigenarmee war eine rasche Vergrößerung der britischen Berufsarmee nach dem Kriegseintritt der westlichen Mächte schwierig ${ }^{25}$. Der Ende 1854 erlassene »Enlistment of Foreigners Act « ermöglichte die Anwerbung von Ausländern »for the duration of the present war « ${ }^{26}$. Danach war den Söldnern eine freie Reise in ihr Heimatland oder zu einem anderen kontinentaleuropäischen Ziel oder aber, als spezieller Anreiz sowohl für politisch Verfolgte als auch Männer aus prekären wirtschaftlichen Verhältnissen, die Überfahrt nach Nordamerika garantiert ${ }^{27}$. War ursprünglich eine Stärke von 5000 Mann angestrebt $^{28}$, so übertrafen die Rekrutierungserfolge alle Erwartungen, indem sich über

23 Porch, The French foreign legion (wie Anm. 7), S. 50-117; Montagnon, La Légion étrangère (wie Anm. 13), S. 29-44.

24 Vgl. TNA, WO 32/7558: Foreign Legion for the Service of Her Britannic Majesty, 1855; ebd., WO 25/3861: Foreign Legions; ebd., WO 32/8342: Formation and disbandment of Swiss Legion; ebd., WO 33/2A: British Foreign Legion; ebd., WO 4/504: Letter Book relating to the Foreign Legion from 11July 1855; ebd., WO 4/505: Letter Book relating to the Foreign Legion from Sept 3 1856; ebd., WO 4/506: Letter-Book of the British Foreign Legion, vol. 3 from 1June 1857; ebd., HO 45/6231: War: Foreign Legion at Shorncliff and Dover; ebd., FO 412/3: British Foreign Legion: Discharge of Soldiers. Correspondence; ebd., FO 412/4: British Foreign Legion. Discharge of Soldiers. Correspondence; ebd., FO 881/574: Foreign Legion. Disbanding. Correspondence, sowie Times, 15.12.1854, 19.12.1854, 6.1.1855, 12.1.1855, 21.7.1855, 10.8.1855, 2.10.1855, 15.10.1855, 26.10.1855, 13.5.1856, 19.6.1856; Die deutsche Fremdenlegion in England (Aus England), Leipzig 1855; Bayley, Mercenaries (wie Anm. 6); Percy, Mercenaries (wie Anm. 4), S. 156-163.

25 Bayley, Mercenaries (wie Anm. 6), S. 35.

26 Vgl. Hansard. House of Commons Debates, 3. series, vol. 136, S. 629-687 und S. 794-898; Hansard. House of Lords Debates, 3. series, vol. 136, S. 429-461.

27 Vgl. TNA, WO 32/8342: Articles of Capitulation for the Formation of a Foreign Legion for the Service of Her Britannic Majesty bzw. WO 33/1 Articles of Capitulation for the formation of a foreign legion for the service of her Britannic Majesty. Ursprünglich standen 7-Jahre-Kontrakte zur Diskussion (vgl. ebd., WO 32/7558: Capitulation on the Formation of a Foreign Legion for the Service of Her Britannic Majesty, 1855).

28 TNA, WO 32/8342: Articles of Capitulation for the Formation of a Foreign Legion for the Service of Her Britannic Majesty. 
15000 Freiwillige der britischen Fremdenlegion anschlossen. Sie wurden in je eine deutsche, italienische und Schweizer Legion eingeteilt und kamen aus den folgenden Ländern ${ }^{29}$ :

Tabelle: Rekrutierte in der British Foreign Legion 1855

\begin{tabular}{|c|c|c|c|c|c|c|c|}
\hline Deutsche & & Italiener & & Schweizer & & Andere & \\
\hline Preußen & 2544 & Piemont & 1719 & Schweiz & 3000 & Polen & 1500 \\
\hline Bayern & 1048 & Lombardei & 837 & & & Belgien & 412 \\
\hline Hannover & 816 & Parma & 218 & & & Niederlande & 376 \\
\hline Baden & 548 & Modena & 114 & & & Ungarn & 72 \\
\hline Holstein & 488 & Toskana & 85 & & & & \\
\hline Hessen & 464 & Rom & 59 & & & & \\
\hline Österreich & 400 & Neapel & 25 & & & & \\
\hline Sachsen & 356 & & & & & & \\
\hline Mecklenburg & 284 & & & & & & \\
\hline Württemberg & 108 & & & & & & \\
\hline Nassau & 56 & & & & & & \\
\hline $\begin{array}{l}\text { Rekrutierte } \\
\text { Total }\end{array}$ & 7112 & $\begin{array}{l}\text { Rekrutierte } \\
\text { Total }\end{array}$ & 3057 & $\begin{array}{l}\text { Rekrutierte } \\
\text { Total }\end{array}$ & 3000 & $\begin{array}{l}\text { Rekrutierte } \\
\text { Total }\end{array}$ & 2360 \\
\hline $\begin{array}{l}\text { Stärke bei } \\
\text { Kriegsende }\end{array}$ & & $\begin{array}{l}\text { Stärke bei } \\
\text { Kriegsende }\end{array}$ & & $\begin{array}{l}\text { Stärke bei } \\
\text { Kriegsende }\end{array}$ & & & \\
\hline $\begin{array}{l}\text { Soldaten und } \\
\text { Unteroffiziere }\end{array}$ & 8665 & $\begin{array}{l}\text { Soldaten und } \\
\text { Unteroffiziere }\end{array}$ & 3535 & $\begin{array}{l}\text { Soldaten und } \\
\text { Unteroffiziere }\end{array}$ & 3073 & & \\
\hline Offiziere & 406 & Offiziere & 127 & Offiziere & 127 & & \\
\hline
\end{tabular}

Quelle: TNA, FO 924/4579: A Map showing the number of Men obtained in 1855 for the British Foreign Legion und ebd., WO 32/8324: Draft on verbal instructions through P.S., 26.4.1856.

29 Vgl. für eine Namensliste der Offiziere der »Swiss Legion« Schweizerische Militär-Zeitung, 22.11.1855. 
Die Einteilung der Belgier, Niederländer und Ungarn ist aus den Quellen nicht ersichtlich. Den Kern der polnischen Fremdenlegionäre bildeten Angehörige der russischen Armee, die im August 1854 bei der französisch-britischen Einnahme der Festung Bomarsund auf den Åland-Inseln in Kriegsgefangenschaft geraten waren $^{30}$. Darüber hinaus führten (recht erfolglose) Versuche der Rekrutierung von Deutsch-Amerikanern zu diplomatischen Spannungen mit den Vereinigten Staaten $^{31}$. Zusätzlich zu diesen europäischen Legionen gab es noch türkische Infanterie- und Kavalleriekontingente unter britischem Kommando mit einer Gesamtstärke von etwa $20000 \mathrm{Mann}^{32}$.

Die geografische Herkunft der britischen Fremdenlegionäre ähnelte derjenigen bei der französischen Fremdenlegion. Allerdings konnten sich die Briten im Unterschied zu den Franzosen nicht auf ein Netz von Rekrutierungsstationen im Inland stützen, sondern entfalteten in relativ kurzer Zeit umfangreiche Aktivitäten vor Ort beziehungsweise in den Hauptquartieren der einzelnen Untereinheiten, das sich für die »German Legion« auf der britisch kontrollierten Insel Helgoland und für die »Swiss Legion« im elsässischen Schlettstadt befand. Die britischen Rekrutierungsbemühungen in weiten Teilen Mittel-, Süd- und Westeuropas wirkten offenbar so verzweifelt, dass sich im Sommer 1855 das Gerücht verbreitete, auch Teile der französischen Fremdenlegion würden an Großbritannien ausgeliehen oder abgetreten ${ }^{33}$.

Mit den Rekrutierungen in Deutschland beauftragte die britische Regierung den bereits erwähnten Baron Richard von Stutterheim, der nach dem Ende des Karlistenkrieges in braunschweigischen, schleswig-holsteinischen und dänischen Diensten gestanden und 1853/54 als Sekretär der mexikanischen Gesandtschaft in Berlin bereits Söldner rekrutiert hatte. Stutterheim schickte mehrere Hundert Rekrutierungsagenten $\operatorname{los}^{34}$. In Italien oblagen die Rekrutierungen einem »Board of Officers « bestehend aus dem britischen General Percy und zwei italienischen Obristen mit Erfahrung in verschiedenen südeuropäischen Armeen $^{35}$. In der Schweiz, wo der Abschluss neuer Militärkapitulationen mit dem Ausland in der Verfassung von 1848 verboten worden und 1853 die Anwerbung von Schwei-

30 St. Galler Zeitung, 1.6.1855.

31 Vgl. Richard W. van Alstyne, Crampton, Conspirator or Dupe? In: American Historical Review, 41 (1936), S. 492-502; Bayley, Mercenaries (wie Anm. 6), S. 87-94.

32 Hansard. House of Commons Debates, 3. series, vol. 38, S. 1734.

33 Vgl. Journal de Genève, 10.6.1855.

34 William Westphal, Ten Years in South Africa. Only Complete and Authentic History of The British German Legion in South Africa and the East Indies, Chicago, IL 1892, S. 6f.; Courrier du Valais, 7.1.1855; Bayley, Mercenaries (wie Anm. 6), S. 67-70 und S. 80-87.

35 Times, 5.9.1855. Vgl. auch ebd., 6.2.1856 sowie Bayley, Mercenaries (wie Anm. 6), S. 75-78 und S. 94-100. 
zern unter Strafe gestellt worden war, der individuelle Eintritt in fremde Dienste jedoch bis 1859 legal blieb ${ }^{36}$, warb die britische Gesandtschaft ein aus Offizieren der Schweizer Armee bestehendes Organisationskomitee an, dem unter anderem ein Instruktionsoffizier und ein Mitarbeiter des Eidgenössischen Kriegskommissariats und zugleich Auswanderungsagent angehörten und das an der Grenze auf französischem und piemontesischem Boden sowie insgeheim offenbar auch in der Schweiz selbst zahlreiche Rekrutierungsstationen einrichtete ${ }^{37}$.

Der soziale Hintergrund der Freiwilligen dürfte etwa demjenigen bei der französischen Fremdenlegion entsprochen haben, die entgegen ihrem Ruf, ein Sammelbecken von Kriminellen sowie romantischen Aussteigern und Abenteurern mit bürgerlichem oder gar aristokratischem Hintergrund zu sein, bereits im 19. Jahrhundert in erster Linie Arbeitslose, Außenseiter und Arme mit oftmals geringen familiären Bindungen sowie Asylsuchende anlockte ${ }^{38}$. Zwar behauptete die »Times«, unter den von einem »hatred of the Muscovite cause« zur Teilnahme am »great struggle between freedom and absolutism « motivierten Söldnern befänden sich "frequenly men of gentle, and, occasionally, men even of noble birth « ${ }^{39}$ und folgerte eine sich auf britische Quellen stützende Schweizer Zeitung aus dem tadellosen Auftreten der Legionäre bei einer Inspektion durch Königin Victoria »que ce n'est pas parmi les rebuts de la société que le recrutement s'est opéré « ${ }^{40}$. Solche Stimmen versuchten indessen in erster Linie, die starke Opposition gegen die Fremdenlegion in Großbritannien zu widerlegen, die, wie ein deutscher Leser-

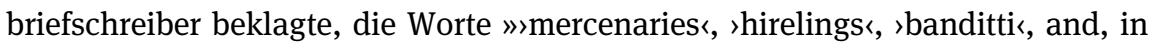

36 Vgl. Johann Jakob Aellig, Die Aufhebung der schweizerischen Söldnerdienste im Meinungskampf des neunzehnten Jahrhunderts, Basel 1954.

37 Vgl. Neue Zürcher Zeitung, 30.12.1854, 24.5.1855, 17.6.1856; St. Galler Zeitung, 31.1.1855, 22.5.1855, 25.5.1855, 8.8.1855; Journal de Genève, 29.7.1855; Courrier du Valais, 31.12.1854, 8.2.1855, 10.6.1855; Botschaft des Bundesrathes an die hohe Bundesversammlung, betreffend die Handhabung des eidgenössischen Werbeverbotes für ausländischen Militärdienst (Vom 13. Juli 1855). In: Bundesblatt 1855, S. 317-359; Henri Ganter, Histoire du service militaire des régiments suisses à la solde de l'Angleterre, de Naples et de Rome, Genf [1901], S. 12; Georg Hoffmann, Die großbritannische Schweizer-Legion im Krimkrieg. Werbung und Schicksal. In: Zeitschrift für Schweizerische Geschichte, 22 (1942), S. 573-595; Edward Attenhofer, British Swiss Legion zur Zeit des Krimkrieges 1854-1856. Wer war der Lenzburger in der Großbritannischen Schweizerlegion? In: Lenzburger Neujahrsblätter 1980, S. 39-51; Bayley, Mercenaries (wie Anm. 6), S. 70-75 und S. 100-106. Für eine Zusammenfassung der medialen Diskussionen in der Schweiz über die Rekrutierungen vgl. Journal de Genève, 10.1.1855.

38 Vgl. z.B. Eduard Blocher, Von der französischen Fremdenlegion. In: Wissen und Leben, $13(1913 / 14)$, S. 327-344, hier: S. 337-339.

39 Times, 10.8.1855.

40 Gazette de Lausanne, 14.8.1855. 
fact, the whole stock of opprobrious epithets in the English language« benutzt hatte ${ }^{41}$.

Die Feststellung des Kommandos der »Swiss Legion«, viele der Angeworbenen hätten »little if anything to lose at home«, gab das Sozialprofil der Mehrheit der Rekrutierten sicherlich korrekter wieder ${ }^{42}$. Ein unbekannter Anteil der Schweizer Freiwilligen hatte zuvor bereits als Söldner in neapolitanischen Diensten gestanden ${ }^{43}$ und bezüglich der in der Stadt Bern Angeworbenen betonte die "Neue Zürcher Zeitung « ausdrücklich den Mangel an Beschäftigungsmöglichkeiten $^{44}$. Die »German Legion« bestand gemäß einem Artikel der »Times« zu einem großen Teil aus »artisans [...] of various classes, and a very large number were agricultural labourers « ${ }^{45}$. Gemäß den Memoiren des Legionärs Wilhelm Westphal aus Hamburg waren die Rekrutierungsagenten in Deutschland am erfolgreichsten in den Hafenstädten,

»where they would find thousands of young men who would do almost anything except work. They would go into some beer saloon where they would treat a number of young men and then describe to them the easy and independent life of a soldier in the British Army, and had no trouble at all to persuade them to join the German Legion, particularly after they got their men under the influence of several glasses of beer. They would, however, give them no time to reconsider their decision ${ }^{46}$."

Mit der vertraglichen Option auf eine Überfahrt nach Nordamerika hatte man es wie erwähnt explizit auf Männer abgesehen, die ein vollständig neues Leben zu beginnen trachteten. In der Tat meldete ein Korrespondent der »Times« im Januar 1855 aus Kiel zu den Rekrutierungsaussichten in Deutschland: »The field is ample, and many who now emigrate would greatly prefer the service of Eng$\operatorname{land}^{47}$. Und im Jahr darauf hielt Colonel Mack von der »German Legion" fest, unter seinen Leuten befänden sich viele, »who are not at all disposed to return to

41 Times, 12.1.1855. Vgl. zu den Einwänden gegen die Fremdenlegion Times, 19.12.1854, und Joseph Howe, Letter to the Right Honourable William E. Gladstone, M.P., by the Hon. Joseph Howe, Being a Review of the Debate on the Foreign Enlistment Bill, and Our Relations with the United States, London 1856.

42 TNA, WO 32/10674: British Swiss Legion, Central Depot Schlestadt, to War Department, April 1856. Vgl. auch Joh[ann] Jak[ob] Romang, Die Englische Schweizerlegion und ihr Aufenthalt im Orient, Langnau 1857, S. 11.

43 Vgl. Ganter, Histoire du service militaire (wie Anm. 37), S. 16 und S. 18.

44 Neue Zürcher Zeitung, 24.5.1855.

45 Times, 1.10.1856.

46 Westphal, Ten Years in South Africa (wie Anm. 34), S. 7.

47 Times, 6.1.1855. 
their homes - or indeed dare not do so, who are anxious to proceed to America ${ }^{48}$. Diese Bemerkung bezog sich hauptsächlich auf Legionäre wie etwa diejenigen aus Preußen, die wegen des Militärdienstes für Großbritannien in ihrer Heimat als Deserteure betrachtet wurden.

Eine nicht unbeträchtliche Zahl der deutschen Freiwilligen waren 48er-Revolutionäre und insbesondere Veteranen des schleswig-holsteinischen Kriegs von 1848 bis $1851^{49}$, die in der Folge in norddeutschen Küstenstädten gestrandet waren. Einige von ihnen hatten 1851/52 als »Brummer« in brasilianischen Diensten im La-Plata-Krieg gekämpft ${ }^{50}$. Hinzu kamen nach Norddeutschland geflüchtete 48er aus Baden und Ungarn ${ }^{51}$. Ein Leutnant der Schweizer Legion titulierte in seinen Memoiren die Kameraden der Deutschen Legion denn auch spöttisch als »vergilbte achtundvierziger Revolutionsmänner«:

»[I]hre Unteroffiziere schienen zum Theil gerade vom Professorenstuhl heruntergestiegen und in die Uniform hineingeschlüpft zu sein, trugen Brillen auf den Nasen und ließen Haare unter dem Tschako herunterfallen, auf die ein Göttinger oder Heidelberger-Burschenschäftler hätte stolz sein können ${ }^{52}$."

Eine gewisse Zahl deutscher Flüchtlinge schaffte es zudem, unter falscher Identität in die »Swiss Legion« aufgenommen zu werden ${ }^{53}$.

Damit erwiesen sich erneut politische Flüchtlinge als ein wichtiges Rekrutierungsreservoir. Nach den Emigrationswellen im Anschluss an die 1830er-Revolutionen und dem polnischen Aufstand von 1830/31 wurden Ende der 1840er Jahre durch die wiederum großteils gescheiterten 1848er-Revolutionen und die polnischen Aufstände von 1846 und 1848 abermals viele Menschen zu Flüchtlingen und manche von ihnen zu Fremdenlegionären. Im Jahre 1850 arbeiteten die schweizerischen und französischen Behörden beim Transfer in der Eidgenossenschaft anwesender 48er-Flüchtlinge in die Fremdenlegion zusammen. Allerdings meldeten sich von den über 12000 Exilanten $^{54}$ nur ein paar Dutzend für den

48 TNA, WO 32/8335: Colonel J. Mack to Lord Panmure, War Department, 23.10.1856.

49 Generell dazu Jan Schlürmann, Die schleswig-holsteinische Armee 1848-1851, Tönning 2004.

50 René Gertz, Brummer. In: Germany and the Americas. Culture, Politics, and History. Ed. by Thomas Adam, Santa Barbara, CA 2005, S. 183.

51 Vgl. auch Die deutsche Fremdenlegion in England (wie Anm. 24), S. 42f.; Romang, Die Englische Schweizerlegion (wie Anm. 42), S. 6 und S. 19 f.

52 Romang, Die Englische Schweizerlegion (wie Anm. 42), S. $19 \mathrm{f}$.

53 Vgl. ebd., S. 12 und S. 108f.; Neue Zürcher Zeitung, 30.8.1856.

54 Albert Portmann-Tinguely und Philipp von Cranach, Flüchtlinge. In: Historisches Lexikon der Schweiz, Bd 4, Basel 2005, S. 565-570, hier S. 566. 
Dienst in Algerien ${ }^{55}$. Nicht umsonst empfahl die piemontesische Regierung im Januar 1855 im Vorfeld des eigenen Kriegseintritts den britischen und französischen Verbündeten, sich bei der Rekrutierung von Fremdenlegionären auf die polnische Emigration zu stützen. Der einflussreiche Exilpole Fürst Adam Jerzy Czartoryski lehnte dies indessen mit Hinweis auf die mangelnde Berücksichtigung nationalpolnischer Interessen durch die Westmächte $\mathrm{ab}^{56}$.

Im Unterschied zur französischen Fremdenlegion, die ab Sommer 1854 gegen Russland kämpfte und dabei 1625 Tote und Verwundete $\mathrm{zu}$ beklagen hatte ${ }^{57}$, gelangte ihr britisches Pendant nicht zum aktiven Einsatz. Zwar trafen einige Regimenter vor dem Friedensschluss vom 30. März 1856 im Orient ein, die Kampfhandlungen waren zu diesem Zeitpunkt aber bereits weitgehend beendet. In den folgenden Monaten setzten intensive Diskussionen über die Zukunft der »British Foreign Legion« ein. Die »Times« bedauerte,

»to see so great an effort, and, we must add, so successful a result, suddenly come to nought. We could whish that a portion at least could be retained [...] In any future war [...] nothing will bring foreigners so thickly to our standards as the knowledge that many by this means have arrived at comfort, independence, even wealth and honour, in our colonies or elsewhere. Whatever is done, care should be taken to make the creation of a new army as easy as can be made ${ }^{58}$."

Als Alternativen zur Auflösung der Einheit und Repatriierung ihrer Angehörigen wurden koloniale Verwendungen und eventuell gar eine Verstetigung der Rekrutierung von Ausländern nach französischem Vorbild oder die Emigration der Legionäre in britische Kolonien diskutiert. Argumente für die erste Variante waren die angeblich positiven Erfahrungen der Franzosen sowie Rekrutierungsprobleme im Inland infolge steigender Lebensstandards der Unterschichten ${ }^{59}$. Allerdings stand im Unterschied zu Algerien, der Hauptbasis der französischen Fremdenlegion, mit Indien das Kerngebiet des britischen Kolonialimperialismus noch nicht unter direkter Regierungsverwaltung. Ein Transfer der »British Foreign

55 Vgl. Kreisschreiben des Schweizerischen Bundesrates an die Regierungen sämmtlicher Stande, betreffend 1) die Aufnahme der Flüchtlinge in die Fremdenlegion in Algerien, 2) die dermalige Anzahl der Flüchtlinge in der Schweiz. In: Bundesblatt 1850, S. 96-99; Neue Zürcher Zeitung, 2.3.1850, 5.3.1850, 9.3.1850, 10.3.1850, 13.3.1850, 5.4.1850; Journal de Genève, 4.3.1850 und 15.3.1850; St. Galler Zeitung, 18.3.1850.

56 Neue Zürcher Zeitung, 15.1.1855.

57 Porch, The French foreign legion (wie Anm. 7), S. 124-128; Montagnon, La Légion étrangère (wie Anm. 13), S. 45-65; John Laffin, The French Foreign Legion, London 1974, S. 60-63.

58 Times, 19.6.1856.

59 Vgl. Times, 13.5.1856. 
Legion« zur in Indien herrschenden »East India Company« wurde 1855/56 zwar angedacht, konkretisierte sich jedoch nicht ${ }^{60}$.

Der Transfer der Legionäre in britische Siedlungskolonien stieß dagegen im Zeichen knapper Kassen nach dem kostspieligen Krimkrieg an finanzielle Grenzen. Eine Anfrage von Colonel Mack bezüglich der Konditionen für Deutsche, die nach Kanada auswandern wollten, beantwortete das "War Department« dahingehend, außer der vertraglich zugesicherten freien Überfahrt könne nichts angeboten werden ${ }^{61}$. Und als eine Gruppe neuseeländischer Kolonisten für die Ansiedlung der Legionäre in Neuseeland lobbyierte, wo es viel Land, aber wenig (und deshalb teure) Arbeitskräfte gebe und zudem dringend Verstärkung in den Konflikten mit den Maori gebraucht würden ${ }^{62}$, wies das »Emigration Office« diesen Vorschlag mit Hinweis auf fehlende Finanzierungsmöglichkeiten zurück ${ }^{63}$.

Interessant erschien daher eine Offerte des argentinischen Generalkonsuls, entlassene Legionäre in Argentinien als Bauern anzusiedeln ${ }^{64}$. Angeboten wurden 10 Morgen Land für Soldaten beziehungsweise 15 Morgen für Unteroffiziere, drei Jahre lang Besoldung und gratis Vieh. In den ersten sechs Jahren hätten die Siedler 20 Prozent des Ertrags aus Ackerbau als Steuern abzuführen, während die Erträge aus der Viehzucht steuerfrei wären; danach sollten Land und Vieh dem Siedler gehören ${ }^{65}$. Militärische Verwendungen der Siedler wie ein Jahrzehnt später von ehemaligen päpstlichen Söldnern, die unter ähnlichen Versprechungen nach Argentinien gelockt, dann aber zum Einsatz im blutigen Tripel-Allianz-Krieg (1864-1870) gepresst wurden ${ }^{66}$, waren in den Konditionen nicht enthalten. Britischerseits schien das Angebot vor allem für italienische Legionäre attraktiv zu sein, von deren Ansiedlung in den eigenen Kolonien man offensichtlich nicht sonderlich begeistert war. In einer Flugschrift an die nach Kriegsende auf Malta stationierten Angehörigen der »Italian Legion ${ }^{67}$ wurde darauf hingewiesen, dass die Regierungen mehrerer italienischer Staaten sich weigerten, Pässe für einige

60 Vgl. TNA, WO 32/8324: Foreign Legion: Suggested use in India, 1856; Courrier du Valais, 10.6.1856.

61 TNA, WO 32/8335: Foreign Legion (British German Legion).

62 TNA, WO 32/8325: New Zealand Colonists Room to Lord Panmure, 9.4.1856.

63 TNA, WO 32/8325: Emigration Office, 4.7.1856.

64 TNA, WO 32/8327: Foreign Legion (Italians): Settlement on discharge.

65 TNA, WO 32/8335: José Buschenthal, Geschäftsführer der Argentinier Republick: EnglischDeutsche Legion.

66 Vgl. Ulrich Lopachers Soldatenleben 1860-70. Hrsg. von Alfred Tobler, Heiden 1912, S. 71; Fabian Brändle, »Maladetti Svizzeri«. Ulrich Lopachers »Soldatenleben« in Italien und Südamerika 1860-1870. In: Krieg und Literatur, 17 (2011), S. 83-95.

67 Vgl. E[rsilio] Michel, Le legione anglo-italiana a Malta, 1856. In: Archivio storico de Malta, 7 (1936), S. 190-212. 
Legionäre auszustellen. Die britische Regierung sei zwar bereit, diese Männer in Kanada oder Südafrika anzusiedeln, das argentinische Angebot sei aber besser; in den britischen Kolonien "gli uomini incontrebbero grandi difficoltà in un clima tanto freddo e tanto differente da quello d'Italia « ${ }^{68}$. Schließlich übersiedelten etwa 160 italienische Legionäre nach Argentinien, wo sie eine bestehende italienische Militärkolonie in Bahía Blanca, einem Außenposten in einem noch weitgehend von Indigenen beherrschten Gebiet, verstärkten ${ }^{69}$.

Für die deutschen Legionäre zeichnete sich bald eine Perspektive als militärische Kolonisten ab. Insbesondere britische Offiziere der »German Legion« lobbyierten für eine solche Lösung. Einer von ihnen schlug in einem Leserbrief die Verwendung in tropischen Kolonien vor, wodurch sowohl Geld als auch Leben britischer Soldaten gespart werden könnten. Kernpunkt seiner Argumentation war das angeblich differierende Trinkverhalten deutscher und britischer Soldaten:

»The German soldier is proverbially sober and tractable. Those qualities are not peculiarly those of Englishmen; and it is a well-known fact that, in our tropical possessions, a large proportion of the troops which guard them die off through the want of sobriety which is the characteristic of the German ${ }^{70}$.»

Nicht in den Tropen, sondern am Kap der Guten Hoffnung sollten indessen die Deutschen angesiedelt werden. Nach einem sich im Sommer 1856 konkretisierenden Plan wurde den Angehörigen der »German Legion« angeboten, sich in der Kapkolonie oder der daran angrenzenden »British Kaffraria« als »Military Settlers« niederzulassen. Die Konditionen umfassten sieben Jahre Dienst, während denen die Kolonisten bei Angriffen von außen eingesetzt werden konnten und an militärischen Übungen teilnehmen mussten. Auch hatten sie, gut viktorianisch, $\mathrm{zu}$ »muster every Sunday for Church parade«. Im Gegenzug erhielten sie Land, auf dem sie ein Haus zu bauen verpflichtet waren, sowie den freien Familienmitzug gewährt ${ }^{71}$. Der Plan stand in einer langen Tradition, angefangen mit den

68 TNA, WO 32/8335: Legione Anglo-Italica.

69 Vgl. Times, 9.7.1856; Bayley, Mercenaries (wie Anm. 6), S. 131-133.

70 Times, 13.5.1856.

71 TNA, WO 32/8331: Report from Major Grant to the Right Honourable Lord Panmure, 14.7.1856; ebd., WO 43/972: Conditions for the Formation of a Military Settlement in British South Africa, War Department, 24.9.1856. Vgl. auch ebd., WO 32/8326: Foreign Legion: Settlement in South Africa; ebd., WO 32/8328: Settlement of British/German legion in Cape of Good Hope. Scale of allowances for building of houses; ebd., WO 32/8329: Conditions for formation of a military colony in British South Africa for members of British/German legion; ebd., WO 32/8330: Conditions for formation of a military colony in British South Africa for members of British/German legion: Memorandum on location; ebd., WO 32/8332: General plan for location of German legion 
antiken Militärkolonien Alexander des Großen und besonders des römischen Imperiums, über die Ansiedlung serbischer und walachischer Wehrbauern an der habsburgischen Militärgrenze gegen das Osmanische Reich seit dem 16. Jahrhundert bis hin zu den ab 1816 eingerichteten russischen Militärsiedlungen (voennye poselenija), die ironischerweise just im selben Jahr definitiv aufgelöst wurden, als die deutschen »Military Settlers« am Kap eintrafen ${ }^{72}$.

Für die Angehörigen der »Swiss Legion« stellten sich ähnliche Fragen. Ein Schreiben des Hauptquartiers in Schlettstadt an das »War Department « vom April 1856 empfahl indessen, die Einheit aufzulösen. Von einer Verwendung in den Kolonien sei aus verschiedenen Gründen abzusehen. Kanada sei »a Colony much sought after by our own Population for the purpose of Emigration \& I venture to suggest that sending Foreign Troops there would be injurious to the prospects of our own Countrymen«. Gegen eine Ansiedlung in Südafrika zusammen mit den Deutschen sprächen dagegen politische Gründe: »The Swiss although brave soldiers are exceedingly Republican in all their ideas, - \& I fear would easily corrupt the Germans, when instead of a Safeguard to our Frontier they would become a Scourge. « Australien schließlich eigne sich nicht »as the men would undoubtedly desert immediately « ${ }^{73}$.

Effektiv kehrte die Mehrheit der Schweizer Legionäre in ihre Heimat zurück. 400 bis 800 von ihnen nahmen aber das Recht auf freie Überfahrt nach Kanada wahr, teilweise mit der Absicht, von dort in die Vereinigten Staaten einzureisen, und 80 weitere gingen in die Kapkolonie. 240 Schweizer und eine unbekannte Zahl von Deutschen folgten dem Ruf eines nach Portsmouth entsandten Rekrutierungsagenten der niederländischen Regierung und begaben sich in Kolonialdienste in Batavia. Einer kleinen Zahl von Deutschen und Schweizern schließlich gelang die Aufnahme in britische Linienregimenter ${ }^{74}$.

Die Ansiedlung ehemaliger Legionäre in Südafrika beschränkte sich also weitgehend auf Deutsche. Insgesamt meldeten sich etwa 3000 Freiwillige, also mehr als ein Drittel der »German Legion«, denen Südafrika vorgestellt worden

in S Africa; ebd., WO 32/8333: Conditions for settlement of German legion in S Africa: Payment of gratuity; ebd., WO 32/8334: Conditions for settlement of German legion in S. Africa: Payment of gratuity, Advance of pay and rates of pay offered; Times, 29.8.1856, 13.9.1856.

72 Vgl. Das Ausland - Ein Tagblatt für Kunde des geistigen und sittlichen Lebens der Völker, 10.12.1830; Paul Jaillet, Essai historique et critique sur la colonisation militaire, Paris 1903; Jakob Amstadt, Die k.k. Militärgrenze 1522-1881, Würzburg 1969.

73 TNA, WO 32/10674: British Swiss Legion, Central Depot Schlestadt, to War Department, April 1856.

74 Romang, Die Englische Schweizerlegion (wie Anm. 42), S. 107-110; Ganter, Histoire du service militaire (wie Anm. 37), S. 25; Hoffmann, Die großbritannische Schweizer-Legion (wie Anm. 37), S. 593. 
war als »a regular paradise where the finest fruits were growing wild, and where the weather was continual summer « und wo, "after we had worsted the negroes [...] all of the beautiful land would be our property « ${ }^{75} .2400$ von ihnen wurden akzeptiert $^{76}$ und in drei Korps zusammen mit ihren Familien nach Südafrika verschifft, wo sie bis Februar 1857 eintrafen. Ihre Ansiedlung sollte das britische Territorium an seiner Ostgrenze absichern, ein Gebiet, das kurz zuvor durch eine Hungersnot unter den Xhosa stark entvölkert worden war ${ }^{77}$. Nachdem die »Military Settlers« die Ortschaften New King William's Town, Keiskammahook, Brunswick, Hamburg, Breitenbach, Berlin, Charlottenburg, Potsdam, Cambridge, Hannover, Marienthal, Wiesbaden, Frankfurt, Ulsen und Stutterheim gegründet hatten $^{78}$, startete die britische Regierung in Norddeutschland eine Anwerbekampagne für Bauern, von denen sich in den Jahren 1858/59 etwa 2000 bis 3000 in und um die neuen Ortschaften ansiedelten ${ }^{79}$.

Die Anwesenheit der deutschen Legionäre in Südafrika ermöglichte es der britischen Regierung, während des großen indischen Aufstandes von 1857/58 die meisten britischen Truppen vom Kap nach Indien zu verlegen. Schließlich wurden auch unter den deutschen Legionären Freiwillige für Indien rekrutiert. Etwa 1100 von ihnen gingen Ende 1858 nach Indien, wo sie allerdings erst nach der Niederschlagung des Aufstandes eintrafen. Ein Teil von ihnen starb bald an

75 Westphal, Ten Years in South Africa (wie Anm. 34), S. 13f.

76 TNA, WO 32/8336: Memorandum for Lord Panmure, 5.11.1856.

77 Vgl. Jörg Fisch, Geschichte Südafrikas, 2. Aufl., München 1991, S. 151.

78 Westphal, Ten Years in South Africa (wie Anm. 34), S. 51-55. Vgl. auch TNA, WO 12/10789: 1 German Settlers: Includes muster books during embarkation and transportation (e.g on board Stamboul), Oct 1856-Feb 1857, and following arrival in the Cape, to March 1858; ebd., WO 12/ 10790: $1^{\text {st }}$ and $2^{\text {nd }}$ German Settlers: Includes musters for $1^{\text {st }}$ regiment (Apr-Sept 1858) and $2^{\text {nd }}$ regiment (Apr 1858-Mar 1859); ebd., WO 12/10791: $2^{\text {nd }}$ German Settlers: Includes muster books during transportation (e.g on board Sultana), Nov 1856-Feb 1857, and following arrival in the Cape, to March 1858; ebd., WO 12/10792: $2^{\text {nd }}$ German Settlers: Includes muster books for April 1859-March 1860; ebd., WO 12/10793: $2^{\text {nd }}$ and $3^{\text {rd }}$ German Settlers: Includes musters for $2^{\text {nd }}$ regiment (Apr-Aug 1860) and $3^{\text {rd }}$ regiment (Apr 1860-Mar 1861); also quarterly pay lists for commissioned officers of the Corps of German Military Settlers, April-Sept 1861; ebd., WO 12/ 10794: $3^{\text {rd }}$ German Settlers: includes detachment embarked on board the Abyssinian, Vulcan, and Mersey, dates to Feb 1857, and $3^{\text {rd }}$ regiment (including attached Cavalry), March 1857-March 1858; ebd., WO 12/10795: $3^{\text {rd }}$ German Settlers: includes musters (including attached Cavalry) for April 1858-March 1859; ebd., WO 12/10796, $3^{\text {rd }}$ German Settlers: includes musters (including attached Cavalry) for April 1859-March 1860.

79 Westphal, Ten Years in South Africa (wie Anm. 34), S. 60-64; Fisch, Geschichte Südafrikas (wie Anm. 77), S. 151f.; W. Hellberg, Vom Schicksal der Krim-Legion, Windhuk 1945; Emrys Wynn Jones, A Leaf on a turbulant river. Ensign Simner and the British German Legion, 1855-9. In: Military History Journal, 13 (2004), S. 60-65 und S. 106-111; Golf Dornseif, Deutsche Legionäre 1856. »Kaffernpuffer« am Kaffraria Ostkap <www.golf-dornseif.de> (letzter Zugriff 17.11.2012). 
Krankheiten, ein weiterer Teil gelangte in den Dienst regulärer britischer Truppen und ein kleiner Teil trat nach einigen Monaten die Rückreise nach Südafrika an. In den folgenden Jahren wechselten auch die verbliebenen deutschen Legionäre in Südafrika zu einem großen Teil in reguläre britische Einheiten oder quittierten den Dienst, sodass die »British German Legion« im Februar 1861 formal aufgelöst wurde $^{80}$.

Während die französische Fremdenlegion sich im Krimkrieg definitiv als eine Elitetruppe für Einsätze sowohl in Imperial- als auch in europäischen Kriegen etablierte und schon 1859 im sardisch-österreichischen Krieg und dann von 1863 bis 1867 bei der französischen Mexiko-Expedition wieder an zentraler Stelle zum Einsatz gelangte ${ }^{81}$, löste sich ihr britisches Pendant trotz Versuchen einer teilweisen Verstetigung und noch Jahre anhaltenden Gerüchten über neue Rekrutierungen $^{82}$ also rasch wieder auf. Dafür waren neben der in den zeitgenössischen britischen Diskussionen immer wieder angeführten Finanzknappheit auch strukturelle und konzeptionelle Gründe verantwortlich: Erstens besaß die französische Fremdenlegion mit Algerien eine vom Mutterland rasch erreichbare Homebase, von der aus sie sowohl für europäische als auch für zahlreiche koloniale Konflikte schnell verfügbar war, während ihr britisches Pendant einer solchen zugleich zentralen und peripheren Basis entbehrte und in der kurzen Zeit ihres Bestehens teils in England, teils im verbündeten Ausland und teils auf britischen Außenposten stationiert war. Zweitens folgten die beiden Fremdenlegionen bezüglich des Umgangs mit ihrer multinationalen Struktur unterschiedlichen Konzeptionen. Teilten die Briten ihre Truppe in national homogene Legionen ein und verdeutlichten auch dadurch den temporären Charakter der Zugehörigkeit zur britischen Armee, hatten die Franzosen dieses System bereits 1835 zugunsten gemischter Einheiten, des sogenannten amalgame aufgegeben, das grundsätzlich dem später offizialisierten Motto »Legio Patria Nostra« entsprach. Damit verknüpft waren drittens unterschiedliche Angebote an ehemalige Legionäre. Stand am Ende des Dienstes in der französischen Fremdenlegion idealerweise die Aufenthaltsberechtigung im französischen Mutterland oder gar der Erwerb der französischen Staatsbürgerschaft, verfolgten die Briten eine reichlich improvisierte und nicht zuletzt durch Nationalstereotypen geleitete Kolonistenpolitik. Die beiden Konzeptionen ähnelten damit stark den nachmaligen kolonialpolitischen

80 Fisch, Geschichte Südafrikas (wie Anm. 77), S. 151f.

81 Porch, The French foreign legion (wie Anm. 7), S. 137-162; Laffin, The Foreign Legion (wie Anm. 57), S. 64-70; Montagnon, La Légion étrangère (wie Anm. 13), S. 66-80.

82 Vgl. St. Galler Zeitung, 12.4.1856, 12.9.1857, 31.12.1861; Neue Zürcher Zeitung, 12.9.1857 und 28.11.1857. 
Doktrinen von der »assimilation« (im Falle Frankreichs) beziehungsweise der »association« (im Falle Großbritanniens).

\section{Wiederbelebung der Idee einer $» B$ Bitish Foreign Legion« im Zeitalter der Weltkriege}

Im Zeitalter des Hochimperialismus ab etwa 1880, als die französische Fremdenlegion ihre Bestände massiv vergrößerte und als integraler Bestandteil der »Armée d'Afrique« bei nahezu jeder kolonialen Erwerbung Frankreichs eine Rolle spielte, verfügte Großbritannien über keine Fremdenlegion, weder innerhalb noch außerhalb der das Kernstück seiner Kolonialeinheiten darstellenden und nach dem Aufstand von 1857/58 grundlegend reorganisierten »Indian Army « ${ }^{83}$. Das Image der französischen Fremdenlegion in Großbritannien war wohl weniger schlecht als im weit stärker von den Rekrutierungen betroffenen Deutschland ${ }^{84}$, dennoch herrschte auch hier die Auffassung, die Fremdenlegion umfasse »the runaway aristocrats and broken men of half Europe ${ }^{85}$. Die britische Presse berichtete seit den 1890er Jahren regelmäßig über abenteuerliche Erlebnisse in der

83 Vgl. zur »Armée d'Afrique« L’Armée d'Afrique 1830-1962. Ed. par Robert Huré, Paris, Limoges 1977; Anthony Clayton, France, Soldiers and Africa, London 1988; Leland Conley Barrows, L'influence des conquêtes algériennes et coloniales sur l'armée française (1830-1919). In: Le Mois en Afrique, 192/193 (1981/82), S. 97-127, und 194/195 (1982), S. 125-149. Zur »Indian Army«: T[homas] A. Heathcote, The Military in British India. The Development of British Land Forces in South Asia, 1600-1947, Manchester 1995; David Omissi, The Sepoy and the Raj. The Indian Army, 1860-1940, Basingstoke 1994; S[tan] L. Menezes, Fidelity and Honour. The Indian Army from the Seventeenth to the Twenty-First Century, New Delhi 1993; Charles Chenevix Trench, The Indian Army and the King's enemies, 1900-1947, London 1988; Byron Farwell, Armies of the Raj. From the mutiny to independence, 1858-1947, London 1990; Philip A. Mason, A Matter of Honour. An Account of the Indian Army, its Officers and Men, London 1974; Boris Mollo, The Indian Army, Poole 1981; Ian Sumner, The Indian Army 1914-1947, Oxford 2001.

84 Vgl. Eckard Michels, Deutsche in der Fremdenlegion. Mythen und Realitäten 1870-1965, Paderborn [u.a.] 1999 (= Krieg in der Geschichte, 2), S. 55-64 und S. 88-98; Marielouise Christadler, Schreckensbild und Vorbild: Die Fremdenlegion in der deutschen Literatur und Propaganda vor 1914. In: Visions allemandes de la France (1871-1914). Ed. par Helga Abret et Michel Grunewald, Bern [u.a.] 1994, S. 63-77. Im internationalen Vergleich Nicola J. Cooper, The French Foreign Legion. Forging Transnational Identities and Meanings. In: French Studies, 17 (2006), S. 269-284; Christian Koller, Kriminelle Romantiker in der exotischen Hölle. Zur transnationalen Medialisierung der französischen Fremdenlegion. In: Saeculum, 63 (2012), S. 247-265.

85 Bristol Mercury, 24.11.1899. 
Fremdenlegion $^{86}$ und vor allem Desertionen ${ }^{87}$, aber auch über Kriminelle in der Legion und kriminell gewordene ehemalige Legionäre ${ }^{88}$. Ab der Zwischenkriegszeit wurde das britische Bild von der französischen Fremdenlegion nachhaltig geprägt durch den in der Folge mehrfach verfilmten Roman »Beau Geste« (1924) des Abenteuerschriftstellers P.C. Wren ${ }^{89}$. Während der beiden Weltkriege verbesserte sich indessen in den britischen Medien das Image der Söldnertruppe des Kriegsverbündeten jeweils schlagartig ${ }^{90}$.

Eine eigene Fremdenlegion war bis in die späten 1930er Jahre kaum ein Thema. Die einzige Ausnahme war bezeichnenderweise das Jahr 1916, als Großbritannien die allgemeine Wehrpflicht einführte und auch (ebenfalls mit französischen Vorbildern argumentierende) Forderungen nach einer intensiveren Rekrutierung in den Kolonien laut wurden ${ }^{91}$. Der konservative Abgeordnete Sir Robert Paterson Houston machte sich im Oktober 1916 für eine Ausdehnung des Gebrauchs von Kolonialtruppen wie auch eine eigene Fremdenlegion stark:

\begin{abstract}
»Let us have real men, even if not white men, men who will fight. I think that my suggestion to have coloured men will appeal to the conscientious objector who objects to fight himself. I do not suppose that he will object to Zulus fighting for him [...] We have in the country many men of military age of Allied nationalities [...] why should we not have a foreign legion and allow these men to enlist in that foreign legion? France for many years has had a foreign legion. The men who enlist in that legion are not Frenchmen. They are Alsatians, Germans, Englishmen, Americans, Russians, men of all nationalities. And there is not a finer fighting force in the world than the French foreign legion". "
\end{abstract}

Weder die Ausdehnung des Einsatzes britischer Kolonialtruppen in Europa noch die Errichtung einer britischen Fremdenlegion sollten aber Realität werden. Die

86 Vgl. z. B. Yorkshire Herald, 17.5.1894; Manchester Guardian, 28.12.1932.

87 Vgl. z. B. Bristol Mercury, 10.8.1895; Times, 16.12.1908, 17.12.1908, 18.12.1908, 19.12.1908, 10.3.1909, 1.3.1911, 2.8.1911, 15.6.1914, 2.6.1928, 4.2.1931, 10.10.1933, 17.10.1933, 31.10.1933, 27.11.1934, 29.12.1937, 23.2.1955, 28.3.1955, 31.3.1959, 7.9.1959, 12.6.1962, 15.8.1962, 20.8.1962, 5.9.1964; Guardian, 24.12.1960, 23.8.1961, 13.6.1962; Manchester Guardian, 20.2.1914, 15.5.1928.

88 Vgl. z.B. Times, 4.2.1920, 11.8.1930, 23.8.1934, 22.8.1938; Observer, 24.10.1926; Manchester Guardian, 28.2.1952.

89 Percival Christopher Wren, Beau Geste, London 1924.

90 Vgl. z.B. Times, 8.9.1916, 7.10.1916, 25.5.1917, 27.7.1940, 26.8.1940; Manchester Guardian, 3.5.1941.

91 Vgl. David Killingray, The Idea of a British Imperial African Army. In: Journal of African History, 20 (1979), S. 421-436; Christian Koller, »Von Wilden aller Rassen niedergemetzelt«. Die Diskussion um die Verwendung von Kolonialtruppen in Europa zwischen Rassismus, Kolonialund Militärpolitik (1914-1930), Stuttgart 2001 (= Beiträge zur Kolonial- und Überseegeschichte, 82), S. 158-162.

92 Hansard. House of Commons Debates, 5. series, vol. 86, S. 117f. Vgl. auch ebd., vol. 87, S. 950 und S. $1187 \mathrm{f}$., und vol. 88 , S. $305 \mathrm{f}$. 
verwaltungstechnisch teilweise als »Foreign Legion« bezeichnete, 1917 aufgestellte und 1921 wieder aufgelöste »Jewish Legion«, die aus mehreren Tausend Freiwilligen vor allem aus den Vereinigten Staaten (darunter viele Immigranten aus Russland), Großbritannien und Palästina bestand, hatte einen grundlegend anderen Charakter als etwa die französische Fremdenlegion. Ihre Errichtung war hauptsächlich politisch bedingt und zielte darauf ab, das vermeintlich einflussreiche amerikanische Judentum für die britische Sache zu begeistern. Der militärische Nutzen war dagegen eher nebensächlich ${ }^{93}$. Während Frankreich nach dem Ersten Weltkrieg eine beträchtliche Zahl der »weissen« Exilanten aus Russland in der Fremdenlegion absorbierte, die aufgrund der zahlreichen Kosaken 1922 erstmals ein Kavallerieregiment erhielt ${ }^{94}$, stand dies in Großbritannien nicht zur Debatte.

Diskussionen über die Errichtung einer eigenen Fremdenlegion starteten erst wieder im Jahre 1939, einige Monate vor Beginn des Zweiten Weltkrieges. Wie bei den Ansätzen im 19. Jahrhundert überkreuzten sich dabei militär- und flüchtlingspolitische Überlegungen. Von März bis Juli 1939 brachten verschiedene Parlamentarier das Thema in beiden Häusern auf ${ }^{95}$. Im April forderte der konservative Abgeordnete Alfred Duff Cooper, ein Gegner der Appeasement-Politik, der 1938 aus Protest gegen das Münchner Abkommen als Marineminister zurückgetreten war, in einem Brief an Kriegsminister Leslie Hore-Belisha, angesichts der Vielzahl von kampfbereiten Hitler-Gegnern im britischen Exil sei entweder eine »Foreign Legion« einzurichten oder der Eintritt von Ausländern in die reguläre Armee zu gestatten ${ }^{96}$. Vier Monate später doppelte Norman Angell, langjähriger pazifistischer und dann antifaschistischer Aktivist und Publizist, ehemaliger

93 Vgl. Elias Gilner, War and Hope. A History of the Jewish Legion, New York 1969; Martin Watts, The Jewish Legion and the First World War, New York 2004, sowie TNA, WO 32/11347: Offer to raise Jewish army to conquer Palestine 1914; ebd., WO 32/11348: Proposal by M Jabotinsky to raise corps of Jewish volunteers for service in Palestine 1915; ebd., WO 32/11349: Proposal by Jabotinsky that Zion Mule Corps be converted into a fighting force. Recruitment of foreign Jews for Zion Corps 1915; ebd., WO 32/11350: Policy and legal position as to raising of Jewish Foreign Legion 1915-1916; ebd., WO 32/11351: Proposal to raise battalion of Russian and Polish Jews 1916; ebd., WO 32/11352: Discussion as to formation of Jewish Legion for service in Palestine 1916-1917; ebd., WO 32/11353: Formation of regiment of Jews following proposals by M. Jabotinsky. Designation of battalions raised specially for Jews in Royal Fusiliers.

94 Vgl. TNA, FO 141/816/1: Le Lieut-Colonel Méru, Officier de Liaison, à Monsieur le Maréchal Allenby, Résidence, 27.5.1920; Zinovi Pechkoff, The Bugle Sounds. Life in the Foreign Legion, New York, London 1926, S. 9f.

95 Hansard. House of Commons Debates, 5. series, vol. 344, S. 1374f. und S. 1904f., vol. 345, 1296f., vol. 347, S. 1009f.; Hansard. House of Lords Debates, 5. series, vol. 114, S. 101.

96 TNA, WO 32/10674: Duff Cooper an Leslie Hore-Belisha, 21.4.1939 sowie Journal de Genève, 10.3.1939 und Gazette de Lausanne, 10.3.1939. 
Labour-Abgeordneter und Friedensnobelpreisträger von 1933, in einem Leserbrief in der »Times « nach und beklagte, trotz der Anwesenheit tausender antifaschistischer Flüchtlinge in Großbritannien, «all suggestions for the creation of a Foreign Legion seem to be cursorily rejected ${ }^{97}$.

Tatsächlich war man im »War Office« selbst nach Kriegsausbruch von der Idee nicht begeistert. Mitte September 1939 wurde in einem internen Dokument als »attractive solution« der Flüchtlingsfrage sogar die Idee aufgeworfen »to hand them over to the French, who [i] have the >drill for utilising foreigners and (ii) are likely to be in need of manpower sooner than we are ${ }^{98}$. Im Januar 1940 wurde die Forderung nach einer britischen Fremdenlegion im Unterhaus erneut vorgebracht ${ }^{99}$. Fünf Tage nach Beginn des deutschen Westfeldzuges brachte am 15. Mai 1940 ein Artikel der »Times« das Thema wieder aufs Tapet ${ }^{100}$. Sekundiert wurde der Vorschlag unter anderem von einer Eingabe ans Kriegsministerium und einem Leserbrief der sozialdemokratischen Exilorganisation »Austrian Office«, dies zu einem Zeitpunkt, als die österreichischen Exilanten als »enemy aliens« von Verhaftung und Internierung bedroht waren ${ }^{101}$. Norman Angell pries die Idee einer Fremdenlegion explizit als Alternative zur Internierung an, während andere Stimmen mit der Wehrgerechtigkeit argumentierten ${ }^{102}$. Ein weiterer Leserbriefschreiber forderte, eine Fremdenlegion sollte nebst Flüchtlingen auch weiteren Ausländern offen stehen, und schlug zur Akzeptanzsteigerung eine semantische Korrektur vor:

»If the term >Foreign Legion` sounds distasteful to British ears, why not give it the name of 'Liberty Legion`, which would express its true and universal object ${ }^{103}$.

Ende Mai wurde im Unterhaus die Forderung nach Einrichtung einer Fremdenlegion für Belgier und Niederländer erhoben ${ }^{104}$.

97 Times, 18.7.1939. Vgl. zu Angells Aktivitäten zu dieser Zeit Martin Ceadel, Living the great illusion. Sir Norman Angell, 1872-1967, Oxford 2009, S. 358-376.

98 TNA, WO 32/10674: Minute Sheet No 2, 15.9.1939. Diese Passage ist im Protokoll von Hand durchgestrichen.

99 Hansard. House of Commons Debates, 5. series, vol. 356, S. 353 f.

100 Times, 15.5.1940.

101 Times, 17.5.1940. Vgl. zum weiteren Kontext Immortal Austria? Austrians in Exile in Britain. Ed. by Charmian Brinson, Amsterdam 2007 (= The yearbook of the Research Centre for German and Austrian Exile Studies, 8); Helene Maimann, Politik im Wartesaal. Österreichische Exilpolitik in Großbritannien 1938 bis 1945, Wien 1975.

102 Times, 21.5.1940.

103 Times, 17.5.1940.

104 Hansard. House of Commons Debates, 5. series, vol. 361, S. 397. 
Nach der Kapitulation Frankreichs und der Konstituierung der »Free French Forces « und der Einheiten der polnischen Exilregierung ${ }^{105}$ auf britischem Boden sowie der im Juli 1940 erfolgten Einrichtung mehrerer Royal Air Force Squadrons mit Tschechoslowaken wurden im Parlament und den Medien erneut Forderungen nach einer britischen Fremdenlegion laut, die vor allem deutsche und österreichische Antifaschisten rekrutieren und eventuell auch einen weiblichen Hilfsdienst umfassen sollte ${ }^{106}$. Im November hakte der Labour-Abgeordnete Seymour Cocks nach und fragte an, ob Schritte »to form a foreign legion or international brigade from the European exiles in this country « unternommen würden ${ }^{107}$. Erstmals wurden damit nicht nur die französische Fremdenlegion, sondern auch die antifaschistischen Freiwilligenverbände des Spanischen Bürgerkriegs semantisch zum Vorbild einer britischen Exilantentruppe erhoben. Die Regierung lehnte diese Forderungen aber aus organisatorischen Gründen $\mathrm{ab}^{108}$. Ausländer sollten in bestehende Truppen aufgenommen werden, insbesondere in das im Oktober 1939 gegründete »Auxiliary Military Pioneer Corps«, das hauptsächlich mit Bauund Logistikaufgaben betraut war und in dem in der Folge in der Tat Tausende von deutschen und österreichischen Exilanten Dienst taten ${ }^{109}$.

In der Folgezeit entstanden einige Ausländer-Einheiten. Neben den schließlich über 200000 Mann starken polnischen Einheiten unter britischem Kommando zu nennen sind etwa das tschechoslowakische »11 ${ }^{\text {th }}$ Infantry Battalion« (ab November 1940) und die $" 1^{\text {st }}$ Czechoslovak Independent Armoured Brigade Group« (ab 1943) sowie ab Juli 1944 die »Jewish Brigade«, die allen Juden offen stand, sich aber hauptsächlich im britischen Mandatsgebiet Palästina rekrutier$\mathrm{te}^{110}$. Die Gründung einer von den österreichischen Exilorganisationen wie auch

105 Vgl. z.B. Michael Alfred Peszke, A Synopsis of Polish-Allied Military Agreements During World War Two. In: Military Affairs, 44 (1980), S. 128-134; Michael Alfred Peszke, The Polish Parachute Brigade in World War Two. A Paradigm for the Polish Military in Exile. In: Military Affairs, 48 (1984), S. 188-193; Matthew R. Schwonek, Kazimierz Sosnkowski as Commander in Chief. The Government-in-Exile and Polish Strategy, 1943-1944. In: Journal of Military History, 70 (2006), S. 743-780.

106 Vgl. Hansard. House of Commons Debates, 5. series, vol. 362, S. 602, 1254f., 1268f., 1275, 1278 und S. 1294, vol. 363, S. 582f. und S. 1135f.; Hansard. House of Lords Debates, 5. series, vol. 117, S. 268f.; Times, 10.7.1940 und 13.7.1940.

107 Hansard. House of Commons, 5. series, vol. 365, S. 1861.

108 Times, 21.6.1940.

109 Vgl. Times, 21.5.1940 sowie Helen Fry, The King’s Most Loyal Enemy Aliens. Germans Who Fought for Britain in the Second World War, Stroud 2007.

110 Vgl. Morris Beckman, The Jewish Brigade. An Army With Two Masters, 1944-45, Rockville Centre 1999; Howard Blum, The Brigade. An Epic Story of Vengeance, Salvation, and WWII, New York 2002. 
Alfred Duff Cooper geforderten »Austrian Legion« wurde dagegen von der Regierung auf die lange Bank geschoben und kam nie zustande ${ }^{111}$. Eben so wenig realisierte sich das Postulat einer allgemeinen britischen Fremdenlegion, das ab 1941 wieder aus den Debatten verschwand ${ }^{112}$.

\section{„Displaced Persons« als Reservoir einer antikommunistischen Fremdenlegion?}

Nach dem Ende des Zweiten Weltkrieges tauchte die Idee einer britischen Fremdenlegion schon rasch wieder auf. Erneut überkreuzten sich dabei militärische und migrationspolitische Argumente. Die Anhänger der Idee argumentierten einerseits mit einem weiterhin hohen Truppenbedarf, um das den Globus umspannende »Commonwealth « zusammenzuhalten und zugleich einen Beitrag zur Abwehr des Kommunismus in Europa leisten zu können, und andererseits mit der großen Zahl an Flüchtlingen, die sich weiterhin in Großbritannien selbst sowie in von der britischen Armee besetzten Gebieten Zentraleuropas aufhielten. Insbesondere ging es um die polnischen Soldaten, von denen sich 1945/46 etwa eine Viertelmillion in Großbritannien aufhielten und nur etwas über 100000 bereit waren, in ihre Heimat zurückzukehren. Die Stimmung in der britischen Bevölkerung gegenüber den Polen schlug gegen Kriegsende ins Negative um und die neue Labour-Regierung wollte die Polen möglichst rasch loswerden ${ }^{113}$.

Im Frühjahr 1946 lobbyierten unter anderem die konservativen Abgeordneten John Jarvis und John Archibald Boyd-Carpenter für eine »British Foreign Legion« aus polnischen, jugoslawischen und anderen alliierten Soldaten, die nicht in ihre Heimatländer zurückkehren wollten oder konnten ${ }^{114}$. Auch in der Bevölkerung geisterten solche Ideen herum. Ein pensionierter Kapitän forderte in einem Brief an Außenminister Ernest Bevin die Rekrutierung polnischer Exilanten und schlug die Einrichtung einer »Elite Foreign Legion, officered by soldiers with multilingual ability« vor, die auch der UNO zur Verfügung gestellt werden

111 Vgl. TNA, WO 32/10674: Duff Cooper to Sir James Grigg, War Office, 31.3.1942.

112 Letzte Thematisierung im Parlament am 10.6.1941 (Hansard. House of Commons Debates, 5. series, vol. 372, S. 14).

113 Peter D. Stachura, The Poles in Scotland, 1940-50. In: The Poles in Britain. From Betrayal to Assimilation. Ed. by Peter D. Stachura, London 2004, S. 48-58, hier S. 51-53.

114 Vgl. TNA, FO 371/56642: Ernest Bevin to Sir John Jarvis, M.P., 26.3.1946; Times, 17.8.1946; Manchester Guardian, 15.3.1946; Hansard. House of Commons Debates, 5. series, vol. 420, S. 142, 275-277 und S. 1367. 
könnte ${ }^{115}$. Eine Anfrage des Außen- beim Kriegsministerium, ob mit einer entsprechenden Truppe eine Lösung für die polnischen Exilanten gefunden werden könnte, wurde jedoch abschlägig beantwortet. Eine britische Fremdenlegion, so das »War Office«, könnte höchstens 5000 bis 6000 Mann umfassen und müsste zudem auch andere Nationalitäten aufnehmen ${ }^{116}$. Stattdessen kündigte die Regierung wenige Wochen später die Bildung eines »Polish Resettlement Corps« an, in dem polnische Soldaten unter militärischer Disziplin auf das Zivilleben in Großbritannien vorbereitet werden sollten, und im folgenden Jahr gewährte ihnen der »Polish Settlement Act« die britische Staatsbürgerschaft. Auch in den Parlamentsdebatten um diese Maßnahmen tauchte die Forderungen nach einer britischen Fremdenlegion wieder auf ${ }^{117}$, während die Regierung betonte, das »Resettlement Corps« sei unter keinen Umständen als eine Art Fremdenlegion zu verstehen $^{118}$.

Die Diskussionen um eine eigene Fremdenlegion gingen indessen weiter, fokussierten nun aber mehr auf die osteuropäischen Flüchtlinge in den britisch kontrollierten Gebieten Zentraleuropas. Zur Jahreswende 1946/47 griff das Flüchtlingsdepartement das Thema Fremdenlegion wieder auf. Es argumentierte einerseits mit der absehbaren Truppenknappheit »as Indian troops cease to be available for duties outside India«, andererseits aber vor allem mit dem Bedürfnis, die zahlreichen Flüchtlinge in den britischen Besatzungszonen in feste Strukturen einzubinden:

"It is essential for major political reasons that the refugee problem should be solved as soon as possible [...] But we are now very near the hard core of non-repatriables, the majorities of the refugees at present on our hands are political dissidents who would almost certainly be severly penalised if they returned to their countries and who are in any case determined not to go, while British public opinion would never stand for their forcible repatriation at the point of the bayonet."

Viele von ihnen seien «fighting men, excellent material highly spoken of by the British officers", deshalb sollte die Bildung einer »foreign legion (of, say, 250000 men)« ins Auge gefasst werden ${ }^{119}$. Dieses Projekt einer Truppe, die etwa

115 TNA, FO 371/56642: A.J. Hemmons, Shipmaster, retired, to Ernest Bevin, Foreign Secretary, 20.3.1946.

116 TNA, FO 371/56642: Minutes, 22.3.1946.

117 Hansard. House of Commons Debates, 5. series, vol. 433, S. 441.

118 Hansard. House of Commons Debates, 5. series, vol. 433, S. 1479-1571; Hansard. House of Lords Debates, 5. series, vol. 146, S. 267.

119 TNA, FO 371/66696: Proposal for the formation of a Foreign Legion from non-repatriable refugees: Memorandum (Refugee Department), 2.1.1947. 
50mal so groß sein sollte als was das «War Office» wenige Monate zuvor als realistisch betrachtet hatte und immerhin noch fünfmal so groß wie die französische Fremdenlegion auf ihrem Allzeithöchststand vom Jahre $1940^{120}$, versandete rasch.

Im frühen Kalten Krieg, als sich die britische Militärstrategie unter anderem mit dem Verlust der indischen Armee auseinandersetzen musste, tauchten Forderungen nach einer »British Foreign Legion« sporadisch wieder auf, ohne indessen je ernsthaft Gehör zu finden ${ }^{121}$. 1949 und 1950 etwa plädierte der Labour-Abgeordnete Reginald Paget im Unterhaus mehrfach für eine aus Ostblock-Flüchtlingen, aber seinetwegen auch Ex-Nazis gebildete Fremdenlegion, die die reguläre britische Armee im Nahen Osten oder in Europa entlasten sollte ${ }^{122}$. Auch sein konservativer Kollege Boyd-Carpenter forderte 1950 wieder eine ähnlich zusammengesetzte britische Fremdenlegion, die als Prototyp einer zukünftigen UNOArmee dienen könnte ${ }^{123}$, während der konservative Abgeordnete Alexander Montagu im selben Jahr die Rekrutierung von Deutschen in eine »British Foreign Legion « als akzeptable Alternative zur von ihm abgelehnten deutschen Wiederbewaffnung bezeichnete ${ }^{124}$. 1955 schlug die »Army League« in ihrem Bericht »The Army in the Nuclear Age« eine aus »Displaced Persons « und »unplaceable young men « gebildete britische Fremdenlegion mit Divisionsstärke vor ${ }^{125}$. Im Jahre 1960 wiederholte der konservative Abgeordnete Anthony Kershaw, der im Zweiten Weltkrieg in Tunesien als Verbindungsoffizier mit der französischen Fremdenlegion zusammengearbeitet hatte, diese Forderung ${ }^{126}$. Und noch 1964, zu einem Zeitpunkt, als sich die französische Fremdenlegion aufgrund ihrer Beteiligung am Generalsputsch von Algiers (1961) und dem weitgehenden Ende des französischen Kolonialismus in einer existenziellen Krise befand ${ }^{127}$, forderte ein Leser-

120 Koller, Die Fremdenlegion (wie Anm. 2), S. 36.

121 Vgl. für den weiteren Kontext Dierk Walter, Zwischen Dschungelkrieg und Atombombe. Britische Visionen vom Krieg der Zukunft 1945-1971, Hamburg 2009.

122 Vgl. Times, 11.3.1949 und 21.5.1950.

123 Times, 25.8.1950.

124 Manchester Guardian, 14.9.1950. Vgl. auch Hansard. House of Commons Debates, 5. series, vol. 427, S. 17, vol. 433, S. 1050, 1075 und S. 2068, vol. 435, S. 1853, vol. 441, S. 501, vol. 457, S. 329 und S. 854 , vol. 462, S. 1451, vol. 463, S. 1213, vol. 464, S. 79, vol. 472, S. 1322 und S. 1559-1622, vol. 473, S. 157, vol. 478, S. 207, 624, 738, 1004f. und S. 1200 f., vol. 480, S. 936, vol. 485, S. 748 und S. 764, vol. 489, S. 221f., vol. 539, S. 852, vol. 568, S. 1839, vol. 631, S. 1255, vol. 632, S. 365f.; Hansard. House of Lords Debates, 5. series, vol. 166, S. 784 f., vol. 196, S. 1237.

125 The Army in the Nuclear Age: Report of the Army League Sub-Committee 1955, London 1955. Vgl. auch Times, 12.11.1955; Manchester Guardian, 12.11.1955; Journal de Genève, 19.11.1955.

126 Observer, 4.12.1960.

127 Vgl. Koller, Die Fremdenlegion (wie Anm. 2), S. 37 f. und S. 72 f. 
briefschreiber in der »Times« die Gründung einer »Commonwealth Legion on the lines of the French Foreign Legion « ${ }^{128}$.

Die britischen Diskussionen wurden im Ostblock, gegen den eine britische Fremdenlegion in den meisten Planüberlegungen gerichtet sein sollte, aufmerksam verfolgt. Die ostdeutsche Presse berichtete immer wieder empört darüber ${ }^{129}$ und schrieb etwa von »DPs als Westblock-Kanonenfutter « ${ }^{130}$ oder »Kopfjäger[n] im englischen Sold « ${ }^{131}$. Ende 1948 meldeten ostdeutsche Zeitungen gar, die Briten hätten in Nordafrika aus deutschen Kriegsgefangenen bereits eine 100000 Mann starke Fremdenlegion aufgebaut ${ }^{132}$.

Die Ideen von einer britischen Fremdenlegion waren im frühen Kalten Krieg nicht die einzigen dieser Art. Viel weiter gediehen zeitgleich analoge Pläne in den Vereinigten Staaten, wo von 1946 bis 1955 das Projekt eines »Volunteer Freedom Corps « vorangetrieben wurde ${ }^{133}$. Spiritus Rector der Idee war der republikanische Senator Henry Cabot Lodge Jr. Die Truppe sollte aus »Displaced Persons« gebildet, in Einheiten gemäß der geografischen Herkunft unterteilt und unter USKommando in Deutschland und Österreich ausgebildet und stationiert werden. Nach Ablauf der Dienstzeit sollten die Soldaten in die USA emigrieren können und die amerikanische Staatsbürgerschaft bekommen. Auch hier ergänzten sich also militärische und migrationspolitische Überlegungen.

Das Projekt erhielt insbesondere nach Dwight D. Eisenhowers Amtsantritt im Jahre 1953 Schub. Eisenhower schwebte ähnlich wie einige Jahre zuvor gewissen britischen Planern eine Armee von 250000 antikommunistischen Staatenlosen mit osteuropäischen Wurzeln vor. Er hoffte mit diesem Projekt die westeuropäische Integration $\mathrm{zu}$ fördern, indem die staatenlosen Flüchtlinge integriert und zugleich das Vertrauen in westliche internationale Kooperationen gestärkt werden sollten. Armeeintern wurde das Projekt teilweise auch als willkommene Alternative zur verstärkten Rekrutierung von Afro-Amerikanern gesehen. Der

128 Times, 17.1.1964. Vgl. zur Idee einer »Commonwealth Legion« auch Hansard. House of Commons Debates, 5. series, vol. 632, S. 153.

129 Vgl. Berliner Zeitung, 24.1.1946, 29.9.1949, 22.3.1950, 24.3.1950; Neues Deutschland, 5.11.1948, 25.9.1949, 14.10.1949, 23.3.1950, 23.11.1954; Neue Zeit, 29.9.1949, 22.3.1950 sowie Manchester Guardian, 25.1.1947.

130 Berliner Zeitung, 5.11.1948.

131 Neues Deutschland, 23.11.1954.

132 Neues Deutschland, 18.12.1948; Berliner Zeitung, 19.12.1948.

133 Vgl. dazu: H.W. Brands, A Cold War Foreign Legion? The Eisenhower Administration and the Volunteer Freedom Corps. In: Military Affairs, 52 (1988), S. 7-11; James Jay Carafano, Mobilising Europe's Stateless. America's Plan for a Cold War Army <www.fas.harvard.edu/ hpcws/ carafano.pdf> (letzter Zugriff 24.4.2011); Susan L. Carruthers, Between Camps. Eastern Bloc »Escapees« and Cold War Borderlands. In: American Quarterly, 57 (2005), S. 911-942, hier S. $918 f$. 
negativ konnotierte Begriff »Foreign Legion« wurde im Kontext des »Volunteer Freedom Corps« trotz der augenscheinlichen strukturellen Parallelen zur französischen Fremdenlegion bewusst vermieden. Im Jahre 1951 etwa hielten die »Joint Chiefs of Staff« (JCS), welche die Pläne grundsätzlich unterstützten, fest, »under no conditions« sei es »desirable to form a United States Foreign Legion ${ }^{134}$.

Genau unter dieser Bezeichnung wurden die Pläne indessen in der europäischen Presse diskutiert ${ }^{135}$. Dadurch erlangten sie eine solche Aufmerksamkeit, dass sich bei den amerikanischen Militärbehörden in Deutschland bereits 1951 Anwerbewillige meldeten, die die geplante amerikanische Truppe als Alternative zur französischen Fremdenlegion verstanden ${ }^{136}$. Die westeuropäischen Regierungen standen dem Projekt ablehnend gegenüber und die CIA warnte zudem vor dessen propagandistischer Ausschlachtung durch den Ostblock ${ }^{137}$. In der Tat verfolgte die Presse des sowjetischen Einflussbereichs das Projekt aufmerksam ${ }^{138}$. Das »Neue Deutschland « schrieb etwa von »Landsknechte[n] der Wall Street « ${ }^{139}$ und betonte, es gehe dabei vor allem um die Rekrutierung nichtdeutscher ehemaliger SS-Leute ${ }^{140}$. Die DDR-Presse setzte das Projekt des »Volunteer Freedom Corps« semantisch gleich mit der 1952 im Rahmen einer Europäischen Verteidigungsgemeinschaft (EVG) geplanten europäischen Armee ${ }^{141}$ sowie der Wiederbewaffnung Westdeutschlands ${ }^{142}$, die allesamt unter dem Schlagwort »amerikanische Fremdenlegion« abgehandelt wurden. Schließlich versandete das Projekt Mitte der 1950er Jahre.

Nebst den Forderungen nach einer »British Foreign Legion« fanden sich in Großbritannien wie in anderen westlichen Ländern in der frühen Nachkriegszeit auch Vorschläge für supranationale Einheiten, die dem Modell der französischen

134 Zit. nach Carafano, Mobilising Europe's Stateless (wie Anm. 133), S. 10.

135 Vgl. z.B. Gazette de Lausanne, 30.1.1951 und 3.10.1951; Journal de Genève, 28.1.1952; Allgemeine Schweizerische Militärzeitung, April 1955.

136 Vgl. Schweizerisches Bundesarchiv, E 5330-1/1975/95/98/1954/852: Akte P. Möckli.

137 Central Intelligence Agency: Special Estimate: Communist Reactions to US Establishment of a »Volunteer Freedom Corps«, 13.4.1953 <www.foia.cia.gov> (letzter Zugriff 24.4.2011).

138 Vgl. z. B. Berliner Zeitung, 18.4.1946, 15.12.1948, 12.11.1949, 17.9.1949, 25.7.1950, 13.12.1950, 11.3.1951, 10.4.1952; Neue Zeit, 1.5.1946, 7.5.1946, 19.5.1946, 9.12.1948, 11.2.1951, 11.3.1951; Neues Deutschland, 9.12.1948, 17.9.1949, 25.9.1949, 11.10.1949, 12.10.1949, 9.2.1951, 22.5.1951, 19.6.1951, 13.11.1951.

139 Neues Deutschland, 13.11.1951.

140 Neues Deutschland, 11.10.1949 und 12.10.1949.

141 Beispielsweise Berliner Zeitung, 2.5.1952; Neue Zeit, 26.4.1952.

142 Beispielsweise Neues Deutschland, 12.3.1952; Berliner Zeitung, 30.6.1954. 
oder einer zu gründenden britischen Fremdenlegion folgen sollten. So forderte 1951 ein Leserbriefschreiber der »Times« eine der französischen Fremdenlegion nachempfundene und gegen den Ostblock gerichtete "Atlantic Power Legion« unter amerikanischer Führung, die polnische Exilanten und andere »Displaced Persons« rekrutieren könnte ${ }^{143}$.

Vor allem aber tauchten bis in die 1960er Jahre immer wieder Ideen für eine entsprechend strukturierte UNO-Armee auf, die auch von der Attlee-Regierung unterstützt wurden. Diese sollte über die Blauhelm-Friedenstruppen hinausgehen, wie sie erstmals 1956 in der Suezkrise vom britischen UN-Untergeneralsekretär Brian Urquhart organisiert wurden. Auf Vorschläge, eine UNO-Armee aus einer britischen Fremdenlegion heraus zu entwickeln, habe ich bereits hingewiesen $^{144}$. Ende der 1940er Jahre kamen aus den britischen Streitkräften verschiedentlich Vorschläge für eine stehende UNO-Streitmacht, die professionell und international zusammengesetzt sein sollte. Der Militärpublizist und ehemalige Panzeroffizier Patrick Henry Hamilton Bryan schlug 1947 eine UNO-Armee aus idealistischen und des Esperanto mächtigen Berufssoldaten vor ${ }^{145}$. Auch im Parlament wurden bis in die frühen 1960er Jahre hinein wiederholt solche Ideen geäußert ${ }^{146}$.

Wie stark sich eine solche Streitmacht am Modell der französischen Fremdenlegion orientieren sollte, war indessen umstritten. Während 1958 der »Manchester Guardian« die Perspektive einer künftigen UNO-Armee als »superior Foreign Legion attracting all the most hard-bitten down-and-outs of five continents « ablehnte ${ }^{147}$, bezeichneten im selben Jahr sowohl der ehemalige Fremdenlegionär Anthony Delmayne als auch James Brennan, der während des Zweiten Weltkrieges als britischer Verbindungsoffizier die Fremdenlegion kennen gelernt hatte, dieselbe als probates Vorbild für eine Streitmacht der Vereinten Nationen ${ }^{148}$. Und 1964 forderte auch der konservative Abgeordnete John Tilney im Unterhaus für die UNO eine »individually recruited, permanent, multiracial, multicoloured

143 Times, 2.1.1951.

144 Vgl. TNA: FO 371/56642: A.J. Hemmons, Shipmaster, retired, to Ernest Bevin, Foreign Secretary, 20.3.1946, sowie Times, 25.8.1950.

145 Dierk Walter, Militärische Futurologie in der britischen Militärpublizistik des frühen Kalten Krieges. In: Portal Militärgeschichte, 19.9.2012 <http://portal-militaergeschichte.de/walter_futu rologie> (letzter Zugriff 24.8.2015), in der pdf, S. $14 \mathrm{f}$.

146 Vgl. Hansard. House of Commons Debates, 5. series, vol. 562, S. 1415-1426, vol. 703, S. 1046-1133; Hansard. House of Lords Debates, 5. Serie, vol. 205, S. 150 und S. 169.

147 Manchester Guardian, 12.8.1958.

148 Anthony Delmayne, Sahara Desert Escape, London 1958, S. 93; Manchester Guardian, 18.9.1958. 
force - on the line of the French Foreign Legion « ${ }^{149}$. Im Jahre 1993 sollte dann Brian Urquhart die Idee erneut vorbringen ${ }^{150}$.

All diese britischen Diskussionen, die sich zeitlich mit den quantitativen Höhepunkten der französischen Fremdenlegion in den 1940er und 1950er Jahren ziemlich exakt deckten, kamen mit der Existenzkrise und schließlich radikalen Verkleinerung der französischen Söldnertruppe Mitte der 1960er Jahre weitgehend zum Erliegen. Der Begriff »Foreign Legion« tauchte in der öffentlichen Diskussion im Zusammenhang mit britischen Streitkräften erst nach der Jahrtausendwende wieder auf, nun aber in einem übertragenen Sinne. Nachdem 1998 für Armeebewerber aus den Commonwealth-Staaten die Bedingung eines fünfjährigen Wohnsitzes im Vereinigten Königreich abgeschafft worden war, nahm die Zahl der Ausländer in der britischen Armee rasch zu und stieg bis 2012 auf etwa 12 Prozent, sodass manche Beobachter die britische Armee insgesamt in der Transformation zu einer Fremdenlegion begriffen sahen ${ }^{151}$.

Erneut überkreuzten sich in diesen Diskussionen Militär-, Migrations- und Finanzpolitik: Rekrutierungsschwierigkeiten in der wirtschaftlichen Aufschwungsphase der Blair-Ära führten bei den Spitzen der Rekrutierungsbehörden zu Vorschlägen, die Anwerbung von Ausländern von außerhalb des »Commonwealth« zu ermöglichen, wobei ein weiteres Mal polnische Immigranten im Zentrum der Überlegungen standen und nebst den eigenen Gurkha-Regimentern die französische Fremdenlegion als Vorbild genannt wurde ${ }^{152}$. Die Sparanstrengungen im Gefolge der Finanzkrise ab 2008, gekoppelt mit Bemühungen um eine Verringerung der Immigration, ließen solche Ideen dann obsolet werden und führten zu einer Gegenbewegung: 2009 wurde für einzelne Einheiten ein maximal zulässiger Ausländeranteil von 15 Prozent eingeführt ${ }^{153}$ und 2013 erfolgte die Wiedereinführung der Wohnsitzanforderungen für Bewerber aus dem »Commonwealth «154.

\section{Fazit}

Die Geschichte des modernen Söldnerwesens im Allgemeinen und der eher phantomhaften britischen Fremdenlegionen im Besonderen steht im Schnittpunkt von

149 Times, 8.12.1964.

150 Brian Urquhart, For a UN Volunteer Military Force. In: The New York Review of Books, 10.6.1993.

151 Vgl. Times, 14.11.2005; Sun, 27.12.2007 und 30.9.2013.

152 Vgl. Daily Mail, 19.3.2008; Guardian, 19.3.2008.

153 BBC News, 29.1.2009.

154 Daily Mail, 11.7.2013. 
Militär- und Migrationsgeschichte. Entsprechend überkreuzten sich in den periodisch aufkommenden britischen Diskussionen um eine eigene Fremdenlegion sowohl im 19. als auch im 20. Jahrhundert militär-, kolonial-, flüchtlings- und finanzpolitische Argumente. Militärpolitisch konzentrierte sich das Vereinigte Königreich aufgrund seiner Insellage zumeist auf die Seehoheit und die militärische Kontrolle der Kolonien und bedurfte dadurch nebst den vor Ort rekrutierten Kolonialtruppen nur einer relativ kleinen Berufsarmee, dies im Unterschied zu Frankreich, das neben einer Wehrpflichtigenarmee zur Verteidigung des Mutterlandes sowohl die Fremdenlegion als auch die aus Afrikanern und Asiaten bestehenden Kolonialeinheiten in der kolonialen Sphäre wie auch (früher und massiver als Großbritannien) bei europäischen Konflikten einsetzte ${ }^{155}$. Es ist denn auch kein Zufall, dass ansatzweise Verwirklichungen einer britischen Fremdenlegion im Kontext europäischer Kriege standen, für die das Vereinigte Königreich nur wenig Landstreitkräfte aufbieten konnte, und dass Forderungen nach einer permanenten Fremdenlegion nach dem Zweiten Weltkrieg in aller Regel mit der Doppelbelastung durch die weltweite Verteidigung des »Commonwealth« und den Beitrag zur Bekämpfung des Kommunismus in Europa begründet wurden.

Eine Analogie zur französischen Situation stellte die starke flüchtlingspolitische Komponente der einschlägigen Debatten, die Verknüpfung von militärischer mit politisch und wirtschaftlich motivierter Migration innerhalb Europas und nach Übersee, dar. Das Söldnerwesen galt in beiden Ländern (und darüber hinaus) als migrationspolitisches Steuerungsinstrument par excellence, um Flüchtlinge in eine feste Struktur einzubinden, sie dort zu kontrollieren, für die eigenen Zwecke nutzbar zu machen und außer Landes schaffen zu können, eventuell mit der mittelfristigen Perspektive der (metropolitanen oder kolonialen) Ansiedlung. Das Söldnerwesen war also zugleich militärische Praxis und Element des »social engineering «. Sein häufig improvisierter Charakter, aber auch normative Vorstellungen aus dem Ideenhorizont von Nationalstaatlichkeit und Volkssouveränität schränkten seinen Gebrauchsbereich in der Moderne aber ein.

Während im Frankreich der frühen 1830er Jahre die Koinzidenz eines politischen Umbruchs mit einem starken Zustrom von Flüchtlingen und der anstehenden Eroberung Algeriens eine für die Einrichtung einer Fremdenlegion einmalige Konstellation bot, war in Großbritannien eine vergleichbare Situation nie vor-

155 Vgl. dazu die in Anm. 83 angeführte Literatur, außerdem Myron Joël Echenberg, Colonial conscripts. The Tirailleurs Sénégalais in French West Africa, 1857-1960, Portsmouth 1991; Koller, »Von Wilden aller Rassen niedergemetzelt (wie Anm. 91); Richard S. Fogarty, Race and War in France. Colonial Subjects in the French Army, 1914-1918, Baltimore 2008; Timothy C. Winegard, Indigenous Peoples of the British Dominions and the First World War, Cambridge 2012; Killingray, The Idea of a British Imperial African Army (wie Anm. 91). 
handen. Die wenigen Ansätze zur Verwirklichung einer britischen Fremdenlegion im 19. Jahrhundert entstanden in Situationen, die vorübergehend eine rasche Rekrutierung von Soldaten erforderten. Das Angebot politischer Flüchtlinge und Arbeitsloser war zwar vorhanden und wurde auch aktiv genutzt, war für die Einrichtung der Söldnertruppen indessen nicht ausschlaggebend. Mitte des 20. Jahrhunderts war die Situation dann weitgehend umgekehrt. Forderungen nach Gründung einer britischen Fremdenlegion waren nun primär migrationspolitisch motiviert. Wenn auch im Zeitalter der Weltkriege, der einsetzenden Dekolonisation und des frühen Kalten Krieges militärpolitische Argumente nicht fehlten, so ist das Bestreben, Flüchtlingen im britischen Einflussbereich eine feste Struktur zu bieten und sie wenn immer möglich von den britischen Inseln wegzuschaffen, als Hauptmotivation unübersehbar. Eine mit Frankreich vergleichbare Fremdenlegions-Tradition, die auch finanzpolitische Vorbehalte zu konterkarieren hätte vermögen können, konnte sich so nie herausbilden. Die »British Foreign Legion« blieb weitgehend ein Phantom. 